\title{
A unified approach to the performance analysis of caching systems
}

\author{
Valentina Martina, Michele Garetto, Emilio Leonardi (*)
}

\begin{abstract}
We propose a unified methodology to analyze the performance of caches (both isolated and interconnected), by extending and generalizing a decoupling technique originally known as Che's approximation, which provides very accurate results at low computational cost. We consider several caching policies (including very attractive one, called $k$-LRU), taking into account the effects of temporal locality. In the case of interconnected caches, our approach allows us to do better than the Poisson approximation commonly adopted in prior work. Our results, validated against simulations and trace-driven experiments, provide interesting insights into the performance of caching systems.
\end{abstract}

\section{INTRODUCTION AND PAPER CONTRIBUTIONS}

In the past few years the performance of caching systems, one of the most traditional and widely investigated topic in computer science, has received a renewed interest by the networking research community. This revival can be essentially attributed to the crucial role played by caching in new content distribution systems emerging in the Internet. Thanks to an impressive proliferation of proxy servers, Content Delivery Networks (CDN) represent today the standard solution adopted by content providers to serve large populations of geographically spread users [1]. By caching contents close to the users, we jointly reduce network traffic and improve user-perceived experience.

The fundamental role played by caching systems in the Internet goes beyond existing content delivery networks, as consequence of the gradual shift from the traditional host-to-host communication model to the new host-to-content paradigm. Indeed, a novel Information-Centric Networking (ICN) architecture has been proposed for the future Internet to better respond to the today and future (according to predictions) traffic characteristics [2]. In this architecture, caching becomes an ubiquitous functionality available at each router.

For these reasons it is of paramount importance to develop efficient tools for the performance analysis of large-scale interconnected caches for content distribution. Unfortunately, evaluating the performance of cache networks is hard, considering that the computational cost to exactly analyse just a single LRU (Least Recently Used) cache, grows exponentially with both the cache size and the number of contents [3], [4]. Nevertheless, several approximations have been proposed over the years [4], [5], [6], [7], [8], [9] which can accurately predict cache performance at an affordable computational cost.

The main drawback of existing analytical techniques is their rather limited scope. Indeed, many of them target only specific caching policies (mainly LRU and FIFO) under simplifying traffic conditions (most of previous work relies on the Independent Reference Model [10]), while the analysis of cache networks has only recently been attempted (essentially for LRU) - see related work in Sec. VI

The main contribution of our work is to show that the decoupling principle underlying one of the approximations suggested in the past (the so called Che approximation) has much broader applicability than the particular context in which it was originally proposed (i.e., a single LRU cache under IRM traffic), and can actually provide the key to develop a general methodology to analyse a variety of caching systems.

In particular, in this paper we show how to extend and generalize the decoupling principle of Che's approximation along three orthogonal directions: i) a much larger set of caching algorithms than those analysed so far (under Che's approximation), implementing different insertion/eviction policies (including a multi-stage LRU scheme, LRU with probabilistic insertion, FIFO and RANDOM); ii) more general traffic model than the traditional IRM, so as to capture the effects of temporal locality in the requests arrival process (in particular, we consider a general renewal traffic model for all the above-mentioned caching policies); iii) a more accurate technique to analyse interconnected caches that goes beyond the standard Poisson assumption adopted so far, and permits considering also smart replication strategies (such as leave-copy-probabilistically and leave-copy-down).

Although in this paper we cannot analyse all possible combinations of the above extensions, we provide sufficient evidence that a unified framework for the performance analysis of caching systems is indeed possible under the Che approximation at low computational cost. Our results for the considered systems turn out to be surprisingly good when compared to simulations (model predictions can be hardly distinguished from simulation results on almost all plots).

Furthermore, under the small cache regime (i.e., cache size small with respect to the content catalogue size), which is of special interest for ICN, our expressions can be further simplified, leading to simple closed-form formulas for the cache hit

(*) V. Martina and E. Leonardi are with Dipartimento di Elettronica, Politecnico di Torino, Italy; M. Garetto is with Dipartimento di Informatica, Università di Torino, Italy. 
probability, revealing interesting asymptotic properties of the various caching policies. The insights gained from our models are also (qualitatively) confirmed by trace-driven experiments.

To the best of our knowledge, we are the first to propose a unified, simple and flexible approach that can be used as the basis of a general performance evaluation tool for caching systems.

This paper extends the previous conference version under several respects: i) our modeling approach has been generalized and successfully applied to cache networks with general (mesh) topology; ii) new material concerning the asymptotic behavior of some of the considered caching policies has been added; iii) several parts of have been modified to improve the overall clarity.

\section{SYSTEM ASSUMPTIONS}

\section{A. Traffic model}

We first recall the so-called Independent Reference Model (IRM), which is de-facto the standard approach adopted in the literature to characterize the pattern of object requests arriving at a cache [10]. The IRM is based on the following fundamental assumptions: i) users request items from a fixed catalogue of $M$ object; ii) the probability $p_{m}$ that a request is for object $m$, $1 \leq m \leq M$, is constant (i.e., the object popularity does not vary over time) and independent of all past requests, generating an i.i.d. sequence of requests.

By construction, the IRM completely ignores all temporal correlations in the sequence of requests. In particular, it does not take into account an important feature often observed in real content request traces, and typically referred to as temporal locality: requests for a given content become denser over short periods of time. The important role played by temporal locality, especially its beneficial effect on cache performance, is well known in the context of computer memory architecture [10] and web traffic [11]. Several extensions of IRM have been already proposed to reproduce content temporal locality [10], [11], [12], [13], [14], [15]. The majority of the proposed approaches [10], [11], [12], [13], [15] share with the IRM the following two assumptions: i) the content catalog is fixed; ii) the request process for each content is stationary (typically it is assumed to be either a renewal process or a semi-Markov-modulated Poisson process). Recently [14] a new traffic model, named Shot Noise Model (SNM), has been proposed as a viable alternative to traditional traffic models to capture macroscopic effects related to content popularity dynamics. The basic idea of the SNM is to represent the overall request process as the superposition of a potentially infinite population of independent inhomogeneous Poisson processes (shots), each referring to an individual content. The definition of analytical models for the evaluation of cache performance under the SNM [14], [16], however, is significantly challenging, as discussed in [15], especially when non-LRU caches and networks of caches are analyzed. Moreover, in [15] it has been shown that the performance of caching system under the SNM traffic model can predicted with high accuracy by adopting a fixed-size content catalogue, and modeling the arrival process of each content by a renewal process with a specific inter-request time distribution.

For the above reasons in this paper we will consider the following traffic model which generalizes the classical IRM. The request process for every content $m$ is described by an independent renewal process with assigned inter-request time distribution. Let $F_{R}(m, t)$ be the cdf of the inter-request time $t$ for object $m$. The average request rate $\lambda_{m}$ for content $m$ is then given by $\lambda_{m}=1 / \int_{0}^{\infty}\left(1-F_{R}(m, t)\right) \mathrm{d} t$. Let $\Lambda=\sum_{m=1}^{M} \lambda_{m}$ be the global arrival rate of requests. Note that, by adopting an object popularity law analogous to the one considered by the IRM, we also have $\lambda_{m}=\Lambda p_{m}$.

As a particular case, our traffic model reduces to the classical IRM when inter-arrival request times are independently, exponentially distributed, so that requests for object $m$ are generated according to a homogeneous Poisson process of rate $\lambda_{m}$. In the following, we will refer to our generalized traffic model as renewal traffic.

\section{B. Popularity law}

Traffic models like the IRM (and its generalizations) are commonly used in combination with a Zipf-like law of object popularity, which is frequently observed in traffic measurements and widely adopted in performance evaluation studies [17], [18].

In its simplest form, Zipf's law states that the probability to request the $i$-th most popular item is proportional to $1 / i^{\alpha}$, where the exponent $\alpha$ depends on the considered system (especially on the type of objects), and plays a crucial role on the resulting cache performance [6]. Estimates of $\alpha$ reported in the literature for various kinds of systems range between .65 and 1 [19].

In our work, we will consider a simple Zipf's law as the object popularity law, although our results hold in general, i.e., for any given distribution of object request probabilities $\left\{p_{m}\right\}_{m}$.

\section{Policies for individual caches}

There exists a tremendous number of different policies to manage a single cache, which differ either for the insertion or for the eviction rule. We will consider the following algorithms, as a representative set of existing policies: 


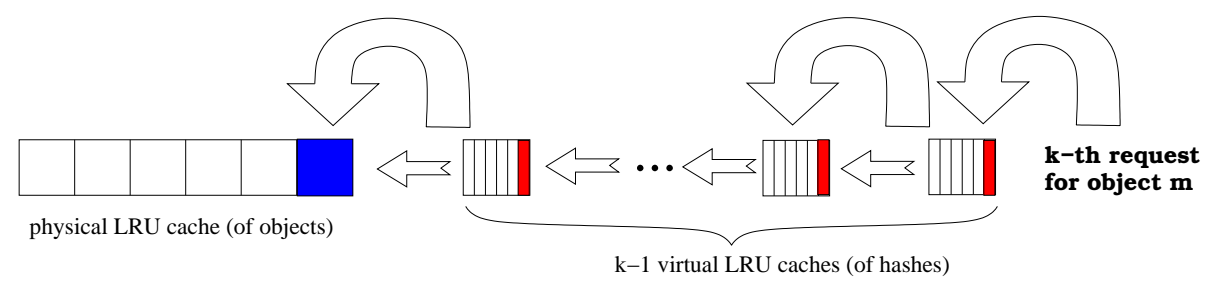

Fig. 1. Illustration of k-LRU policy.

- LFU: the Least Frequently Used policy statically stores in the cache the $C$ most popular contents (assuming their popularity is known a-priori); LFU is known to provide optimal performance under IRM.

- LRU: upon arrival of a request, an object not already stored in the cache is inserted into it. If the cache is full, to make room for a new object the Least Recently Used item is evicted, i.e., the object which has not been requested for the longest time.

- q-LRU: it differs from LRU for the insertion policy: upon arrival of a request, an object not already stored in the cache is inserted into it with probability $q$. The eviction policy is the same as LRU.

- FIFO: it differs from LRU for the eviction policy: to make room for a new object, the item inserted the longest time ago is evicted. Notice that this scheme differs from LRU in this respect: requests finding an object in the cache do not 'refresh' the arrival time associated to it.

- RANDOM: it differs from LRU for the eviction policy: to make room for a new object, a random item stored in the cache is evicted.

- k-LRU: this strategy provides a clever insertion policy by exploiting the following idea: before arriving at the (physical) cache which is storing actual objects, indexed by $k$, requests have to advance through a chain of $k-1$ (virtual) caches put in front of it, acting as filters, which store only object pointers performing caching operations on them (see Fig. (1). Specifically, upon arrival of a request, a content/pointer can be stored in cache $i>1$ only if its pointer is already stored in cache $i-1$ (i.e. the arrival request has produced a hit in cache $i-1$ ). The eviction policy at all caches is LRU. We remark that this policy 1 can be seen as a generalization of the two-stages policy proposed in [20], called there LRU-2Q.

- k-RANDOM: it works exactly like k-LRU, with the only difference that the eviction policy at each cache is RANDOM.

We remark that LRU has been widely adopted, since it provides good performance while being reasonably simple to implement. RANDOM and FIFO have been considered as viable alternative to LRU in the context of ICN, as their hardware implementation in high-speed routers is even simpler. The q-LRU policy and multi-stage caching systems similar to our kLRU have been proposed in the past to improve the performance of LRU by means of a better insertion policy. We have chosen q-LRU in light of its simplicity, and the fact that it can be given an immediate interpretation in terms of probabilistic replication for cache networks (see next section). The main strength of k-LRU, instead, resides in the fact that it requires just one, traffic-independent parameter (the number of caches $k$ ), providing significant improvements over LRU even for very small $k$ (much of the possible gain is already achieved by $k=2$ ).

\section{Replication strategies for cache networks}

In a system of interconnected caches, requests producing a miss at one cache are typically forwarded along one or more routes toward repositories storing all objects. After the request eventually hits the target, we need to specify how the object gets replicated back in the network, in particular along the route traversed by the request. We will consider the following mechanisms [22]:

- leave-copy-everywhere (LCE): the object is sent to all caches of the backward path.

- leave-copy-probabilistically (LCP): the object is sent with probability $q$ to each cache of the backward path.

- leave-copy-down (LCD): the object is sent only to the cache preceding the one in which the object is found (unless the object is found in the first visited cache).

Notice that LCP, combined with standard LRU at all caches, is the same as LCE combined with q-LRU at all caches.

\section{THE CHE APPROXIMATION}

We briefly recall Che's approximation for LRU under the classical IRM [5]. Consider a cache capable of storing $C$ objects. Let $T_{C}(m)$ be the time needed before $C$ distinct objects (not including $m$ ) are requested by users. Therefore, $T_{C}(m)$ is the

\footnotetext{
${ }^{1}$ In the most general case one could individually specify the size of all caches along the chain; however, for simplicity, in this paper we will restrict ourselves to the case in which all caches have the same size (expressed either in terms of objects or pointers), since numerical explorations suggest that no significant performance gains can be obtained by tuning the sizes of individual caches.

${ }^{2}$ More sophisticated insertion policies such as the persistent-access-caching algorithm [21] obtain a filtering effect similar to $k$-LRU but require more parameters which are not easy to set, requiring a-priori knowledge of the traffic characteristics.
} 
cache eviction time for content $m$, i.e., the time since the last request after which object $m$ will be evicted from the cache (if the object is not again requested in the meantime).

Che's approximation assumes $T_{C}(m)$ to be a constant independent of the selected content $m$. This assumption has been given a theoretical justification recently in [6], where it is shown that, under a Zipf-like popularity distribution, the coefficient of variation of the random variable representing $T_{C}(m)$ tends to vanish as the cache size grows. Furthermore, the dependence of the eviction time on $m$ becomes negligible when the catalogue size is sufficiently large. For completeness we wish to remark that an indirect proof of Che's approximation asymptotic validity has been provided earlier in [23] for $\alpha>1$.

The reason why Che's approximation greatly simplifies the analysis of caching systems is because it allows to decouple the dynamics of different contents: interaction among the contents is summarized by $T_{C}$, which acts as a single primitive quantity representing the response of the cache to an object request.

More in detail, thanks to Che's approximation, we can state that an object $m$ is in the cache at time $t$, if and only if a time smaller than $T_{C}$ has elapsed since the last request for object $m$, i.e., if at least one request for $m$ has arrived in the interval $\left(t-T_{c}, t\right]$. Under the assumption that requests for object $m$ arrive according to a Poisson process of rate $\lambda_{m}$, the time-average probability $p_{\text {in }}(m)$ that object $m$ is in the cache is then given by:

$$
p_{\text {in }}(m)=1-e^{-\lambda_{m} T_{c}}
$$

As immediate consequence of PASTA property for Poisson arrivals, observe that $p_{\text {in }}(m)$ represents, by construction, also the hit probability $p_{\text {hit }}(m)$, i.e., the probability that a request for object $m$ finds object $m$ in the cache.

Considering a cache of size $C$, by construction:

$$
C=\sum_{m} \mathbb{I}_{\{m \text { in cache }\}}
$$

After averaging both sides, we obtain:

$$
C=\sum_{m} \mathbb{E}\left[\mathbb{I}_{\{m \text { in cache }\}}\right]=\sum_{m} p_{\text {in }}(m) .
$$

The only unknown quantity in the above equality is $T_{C}$, which can be obtained with arbitrary precision by a fixed point procedure. The average hit probability of the cache is:

$$
p_{\text {hit }}=\sum_{m} p_{m} p_{\text {hit }}(m)
$$

\section{EXTENSIONS FOR SINGLE CACHE}

We will show in the next sections that Che's idea of summarizing the interaction among different contents by a single variable (the cache eviction time) provides a powerful decoupling technique that can be used to predict cache performance also under renewal traffic, as well as to analyze policies other than LRU.

\section{A. LRU under renewal traffic}

The extension of Che's approximation to the renewal traffic model is conceptually simple although it requires some care. Indeed, observe that, under a general request process, we can not apply PASTA anymore, identifying $p_{\text {in }}(m)$ with $p_{\text {hit }}(m)$. To compute $p_{\text {in }}(m)$ we can still consider that an object $m$ is in the cache at time $t$ if and only if the last request arrived in $\left[t-T_{C}, t\right)$. This requires that the age since the last request for object $m$ is smaller than $T_{C}$ :

$$
p_{\text {in }}(m)=\hat{F}_{R}\left(m, T_{C}\right)
$$

where $\hat{F}_{R}(m, t)=\lambda_{m} \int_{0}^{t}\left(1-F_{R}(m, \tau)\right) \mathrm{d} \tau$ is the cdf of the age associated to object- $m$ inter-request time distribution.

On the other hand, when computing $p_{\text {hit }}(m)$, we implicitly condition on the fact that a request arrives at time $t$. Thus, the probability that the previous request occurred in $\left[t-T_{C}, t\right)$ equals the probability that the last inter-request time does not exceed $T_{C}$, yielding:

$$
p_{\text {hit }}(m)=F_{R}\left(m, T_{C}\right)
$$

\section{B. $q$-LRU under IRM and renewal traffic}

We now analyse the q-LRU policy (LRU with probabilistic insertion), considering first the simpler case of IRM traffic. In this case, $p_{\text {in }}(m)$ and $p_{\text {hit }}(m)$ are equal by PASTA.

To compute $p_{\text {in }}(m)$ we exploit the following reasoning: an object $m$ is in the cache at time $t$ provided that: i) the last request arrived at $\tau \in\left[t-T_{C}, t\right)$ and ii) either at $\tau^{-}$object $m$ was already in the cache, or its insertion was triggered by the request arriving at $\tau$ (with probability $q$ ). We obtain:

$$
p_{\text {hit }}(m)=p_{\text {in }}(m)=\left(1-e^{-\lambda_{m} T_{C}}\right)\left[p_{\text {in }}(m)+q\left(1-p_{\text {in }}(m)\right)\right]
$$


Solving the above expression for $p_{\text {in }}(m)$, we get:

$$
p_{\text {hit }}(m)=p_{\text {in }}(m)=\frac{q\left(1-e^{-\lambda_{m} T_{C}}\right)}{e^{-\lambda_{m} T_{C}}+q\left(1-e^{-\lambda_{m} T_{C}}\right)}
$$

Under renewal traffic, $p_{\text {in }}(m)$ and $p_{\text {hit }}(m)$ differ by the same token considered for LRU. Repeating the same arguments as before, we get:

$$
p_{\text {hit }}(m)=F\left(m, T_{C}\right)\left[p_{\text {hit }}(m)+q\left(1-p_{\text {hit }}(m)\right)\right]
$$

which generalizes (4). The age distribution must be instead used to compute $p_{\text {in }}(m)$ :

$$
p_{\text {in }}(m)=\hat{F}\left(m, T_{C}\right)\left[p_{\text {hit }}(m)+q\left(1-p_{\text {hit }}(m)\right)\right]
$$

Regarding the q-LRU policy, Che's approximation allows to establish the following interesting property as $q \rightarrow 0$, whose proof is reported in Appendix $\mathrm{A}$ (IRM case) and $\mathrm{A}$ (non-IRM case).

Theorem 4.1: The q-LRU policy tends asymptotically to LFU as the insertion probability goes to zero both under IRM and under renewal traffic under the following conditions: for any $m_{1}$ and $m_{2}$ with $\lambda_{m_{1}}<\lambda_{m_{2}}$ either $\lim _{t \rightarrow \infty} \frac{1-F\left(m_{1}, t\right)}{1-F\left(m_{2}, t\right)}=\infty$ or a $T$ can be found such that $1-F\left(m_{1}, T\right)>0$ and $1-F\left(m_{2}, T\right)=0$.

Remark: Note that the above condition is satisfied whenever $F(m, t)$ has an exponential tail, i.e., $F(m, t) \approx e^{-\alpha_{m} t}$ with parameter $\alpha_{m}$ monotonically dependent on the average rate $\lambda_{m}$; instead, it is not satisfied whenever distributions $F(m, t)$ are power-law, i.e., $F(m, t) \approx\left(\alpha_{m} t\right)^{-k}$.

\section{RANDOM and FIFO}

The decoupling principle can be easily extended to RANDOM/FIFO caching policies by reinterpreting $T_{C}(m)$ as the (in general random) sojourn time of content $m$ in the cache. In the same spirit of the original Che's approximation, we assume $T_{C}(m)=T_{C}$ to be a primitive random variable (not any more a constant) whose distribution does not depend on $m$.

Under IRM traffic the dynamics of each content $m$ in the cache can be described by an M/G/1/0 queuing model. Indeed observe that object $m$, when not in the cache, enters it according to a Poisson arrival process, then it stays in the cache for a duration equal to $T_{C}$, after which it is evicted independently of the arrival of other requests for content $m$ during the sojourn time.

The expression of $p_{\text {in }}(m)$ and $p_{\text {hit }}(m)$ can then be immediately obtained from Erlang-B formula (exploiting PASTA):

$$
p_{\text {hit }}(m)=p_{\text {in }}(m)=\lambda_{m} \mathbb{E}\left[T_{C}\right] /\left(1+\lambda_{m} \mathbb{E}\left[T_{C}\right]\right)
$$

Notice that we still employ (2) to compute $\mathbb{E}\left[T_{C}\right]$.

As immediate consequence of Erlang-B insensitivity property to the distribution of service time, we conclude that,

Proposition 1: Under IRM traffic, the performance of RANDOM and FIFO (in terms of hit probability) are the same.

This result was originally obtained formally by Gelenbe [24] using a totally different approach that does not resort to Che's approximation.

Note that, under FIFO policy, we can assume $T_{C}$ to be a constant, in perfect analogy to LRU. Indeed, $T_{C}$ is still equal to the time needed to observe the requests for $C$ distinct objects arriving at the cache. On the other hand, under RANDOM policy, it is natural to approximate the sojourn time of an object in the cache with an exponential distribution. Indeed, under RANDOM an object is evicted with probability $1 / C$ upon arrival of each request for an object which is not in the cache.

Under renewal traffic the dynamics of each object under FIFO and RANDOM can be described, respectively, by a G/D/1/0 and a G/M/1/0 queuing model. Observe that, under general traffic, the performance of FIFO and RANDOM are not necessarily the same.

We now show how the RANDOM policy can be analysed, under renewal traffic, employing basic queuing theory. Probability $p_{\text {hit }}$ can be obtained as the loss probability of the G/M/1/0 queue. Simply put, the hit probability $p_{\text {hit }}(m)$ of a given content $m$ equals the probability that the content has not been evicted before the arrival of the next request for content $m$. Having approximated the sojourn time in the cache by an exponential distribution, we can easily compute:

$$
p_{\text {hit }}(m)=\int_{0}^{\infty} e^{-r / \mathbb{E}\left[T_{C}\right]} \mathrm{d} F_{R}(r)=M_{R}\left(m,-1 / \mathbb{E}\left[T_{C}\right]\right)
$$

where $M_{R}(m, \cdot)$ is the moment generating function of object- $m$ inter-request time.

Probability $p_{\text {in }}(m)$ can also be obtained exploiting the fact that the dynamics of a G/M/1/0 system are described by a process that regenerates at each arrival. On such a process we can perform a standard cycle analysis as follows (we drop the dependency of random variables on $m$ to simplify the notation). We denote by $T_{\text {cycle }}$ the duration of a cycle (which corresponds to an inter-request interval). Observe that, by construction, the object is surely in the cache at the beginning of a cycle. Let $\tau$ be the residual time spent by the object in the cache, since a cycle has started, and $T_{\mathrm{ON}}$ be the time spent by the object in the cache within a cycle. 


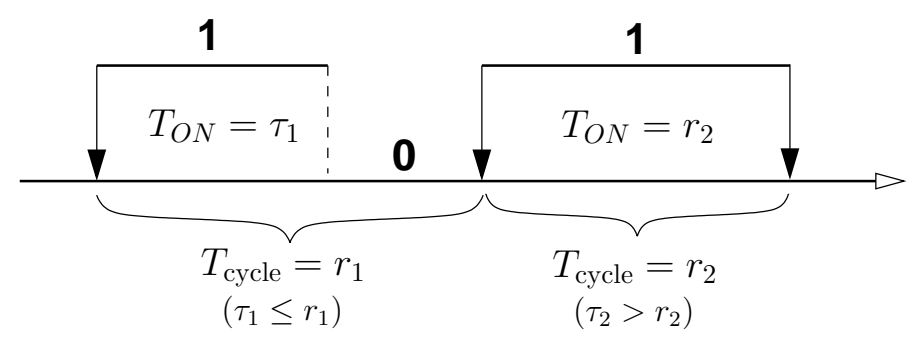

Fig. 2. Illustration of the cycle analysis used for deriving 8. Vertical arrows represent incoming requests for the content.

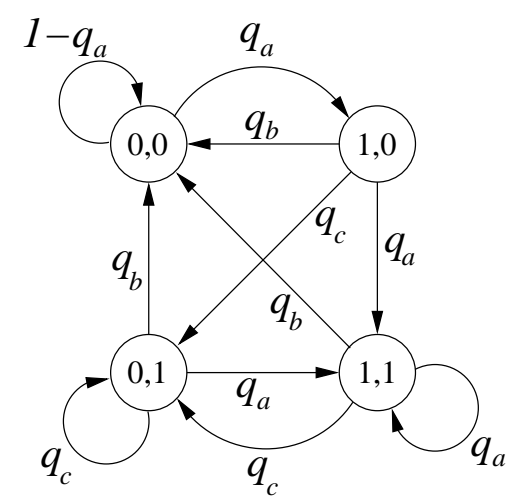

Fig. 3. DTMC describing the dynamics of an object in 2-LRU, sampled at request arrival times

By definition, $T_{\mathrm{ON}}=\min \left\{\tau, T_{\text {cycle }}\right\}$. Thus, by standard renewal theory we have $p_{\text {in }}(m)=\mathbb{E}\left[T_{\mathrm{ON}}\right] / \mathbb{E}\left[T_{\text {cycle }}\right]$. Figure 2 illustrates the two cases that can occur, depending on whether the object is evicted or not before the arrival of the next request. Now, we know that $\mathbb{E}\left[T_{\text {cycle }}\right]=1 / \lambda_{m}$. For $\mathbb{E}\left[T_{\mathrm{ON}}\right]$, we obtain:

$$
\begin{aligned}
\mathbb{E}\left[T_{\mathrm{ON}}\right]=\int_{0}^{\infty}\left(\mathbb{E}\left[T_{\mathrm{ON}} \cdot \mathbb{I}_{\tau \leq r} \mid T_{\text {cycle }}=r\right]+\mathbb{E}\left[T_{\mathrm{ON}} \cdot \mathbb{I}_{\tau>r} \mid T_{\text {cycle }}=r\right]\right) \mathrm{d} F_{R}(r)= \\
=\int_{0}^{\infty}\left(\int_{0}^{r} \frac{x}{\mathbb{E}\left[T_{C}\right]} e^{-x / \mathbb{E}\left[T_{C}\right]} \mathrm{d} x+r e^{-r / \mathbb{E}\left[T_{C}\right]}\right) \mathrm{d} F_{R}(r)
\end{aligned}
$$

In the end, we get $p_{\text {in }}(m)=\lambda_{m} \mathbb{E}\left[T_{C}\right]\left(1-M_{R}\left(m,-1 / \mathbb{E}\left[T_{C}\right]\right)\right)$.

\section{2-LRU}

We now move to the k-LRU strategy, considering first the simple case of $k=2$. For this system, we derive both a rough approximation based on an additional simplifying assumption (which is later used to analyse the more general k-LRU) and a more refined model that is based only on Che's approximation. For both models we consider either IRM or renewal traffic.

Let $T_{C}^{i}$ be the eviction time of cache $i$. We start observing that meta-cache 1 behaves exactly like a standard LRU cache, for which we can use previously derived expressions. Under IRM, $p_{\text {in }}(m)$ and $p_{\text {hit }}(m)$ (which are identical by PASTA) can be approximately derived by the following argument: object $m$ is found in cache 2 at time $t$ if and only if the last request arrived in $\tau \in\left[t-T_{C}^{2}, t\right)$ and either object $m$ was already in cache 2 at time $\tau^{-}$or it was not in cache 2 at time $\tau^{-}$, but its hash was already stored in meta-cache 1 . Under the additional approximation that the states of meta-cache 1 and cache 2 are independent at time $\tau^{-}$, we obtain:

$$
p_{\text {hit }}(m)=p_{\text {in }}(m) \approx\left(1-e^{\left.-\lambda_{m} T_{C}^{2}\right)}\right)\left[p_{\text {hit }}(m)+\left(1-e^{-\lambda_{m} T_{C}^{1}}\right)\left(1-p_{\text {hit }}(m)\right)\right]
$$

Observe that the independence assumption between cache 2 and meta-cache 1 is reasonable under the assumption that $T_{C}^{2}$ is significantly larger than $T_{C}^{1}$ (which is typically the case when the two caches have the same size). Indeed, in this case the states of cache 2 and meta-cache 1 tends to de-synchronize, since an hash is expunged by meta-cache 1 before the corresponding object is evicted by cache 2, making it possible to find an object in cache 2 and not in meta-cache 1 (which otherwise would not be possible if $T_{C}^{1} \geq T_{C}^{2}$ ).

An exact expression for $p_{\text {hit }}(m)$ (under Che's approximation) that does not require any independence assumption can be derived observing that the dynamics of object $m$ in the system, sampled at request arrivals, can be described by the four states Discrete Time Markov Chain (DTMC) represented in Fig. 3, where each state is denoted by a pair of binary variables 


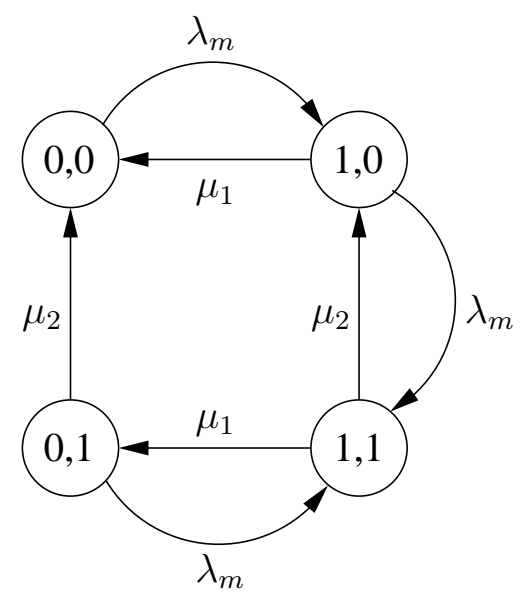

Fig. 4. CTMC describing the dynamics of an object in 2-RANDOM. We denoted $\mu_{1}=1 / T_{C}^{1}, \mu_{2}=1 / T_{C}^{2}$.

indicating the presence of object $m$ in meta-cache 1 and cache 2, respectively. Solving the DTMC, we get:

$$
p_{\text {hit }}(m)=p_{\text {in }}(m)=1-\frac{\left(1+q_{a}\right) q_{b}}{q_{a}+q_{b}}
$$

with $q_{a}=1-e^{-\lambda_{m} T_{C}^{1}}, q_{b}=e^{-\lambda_{m} T_{C}^{2}}$ and $q_{c}=1-\left(q_{a}+q_{b}\right)$.

The extension to renewal traffic can be carried out following the same lines as before. Under the additional independence assumption between the two caches, we obtain:

$$
\begin{aligned}
p_{\text {hit }}(m) & \approx F_{R}\left(m, T_{C}^{2}\right)\left[p_{\text {hit }}(m)+F_{R}\left(m, T_{C}^{1}\right)\left(1-p_{\text {hit }}(m)\right)\right] \\
p_{\text {in }}(m) & \approx \hat{F}_{R}\left(m, T_{C}^{2}\right)\left[p_{\text {hit }}(m)+F_{R}\left(m, T_{C}^{1}\right)\left(1-p_{\text {hit }}(m)\right)\right]
\end{aligned}
$$

Also the refined model can be generalized to renewal traffic, observing that object- $m$ dynamics in the system, sampled at request arrivals (i.e., logically just before a request arrival), are still described by a Markov Chain with exactly the same structure as in Fig. 3 (only the expressions of transition probabilities change in an obvious way). Thus we obtain:

$$
p_{\text {hit }}(m)=1-\frac{\left(1+q_{a}\right) q_{b}}{q_{a}+q_{b}}
$$

with $q_{a}=F\left(m, T_{C}^{1}\right)$ and $q_{b}=1-F\left(m, T_{C}^{2}\right)$

To compute $p_{\text {in }}(m)$ we can resort to a cycle analysis, whose details are reported in Appendix $\mathrm{A}$

\section{E. $k-L R U$}

Previous expressions obtained for 2-LRU (under the independence assumption between caches) can be used to iteratively compute the hit probabilities of all caches in a k-LRU system. For example, under IRM, we can use (95) to relate the hit probability of object $m$ in cache $i, p_{\text {hit }}(i, m)$, to the hit probability $p_{\text {hit }}(i-1, m)$ of object $m$ in the previous cache, obtaining:

$$
p_{\text {hit }}(i, m)=p_{\text {in }}(i, m) \approx\left(1-e^{\left.-\lambda_{m} T_{C}^{i}\right)}\right)\left[p_{\text {hit }}(i, m)+\left(p_{\text {hit }}(i-1, m)\right)\left(1-p_{\text {hit }}(i, m)\right)\right]
$$

The generalization to renewal traffic is straightforward.

At last, for large $k$ we can state:

Theorem 4.2: According to (11) $k$-LRU tends asymptotically to LFU as $k \rightarrow \infty$ under IRM and renewal traffic, as long as the support of the inter-request time distribution is unbounded and for any $m_{1}$ and $m_{2}$, with $\lambda_{m_{1}}<\lambda_{m_{2}}$, it holds $\lim _{t \rightarrow \infty} \frac{1-F\left(m_{1}, t\right)}{1-F\left(m_{2}, t\right)}>1$.

The proof is reported in Appendix $\AA$

\section{F. $k-R A N D O M$}

Also k-RANDOM can be analysed under Che's approximation assuming exponential sojourn times in the caches. As an example, the dynamics of an object in 2-RANDOM (under IRM traffic) are described by the simple four-states continuous time Markov chain depicted in Fig. 4. More in general, k-RANDOM can be exactly analyzed by solving a continuous time Markov chain with $2^{k}$ states. We omit the details of such standard analysis here. 

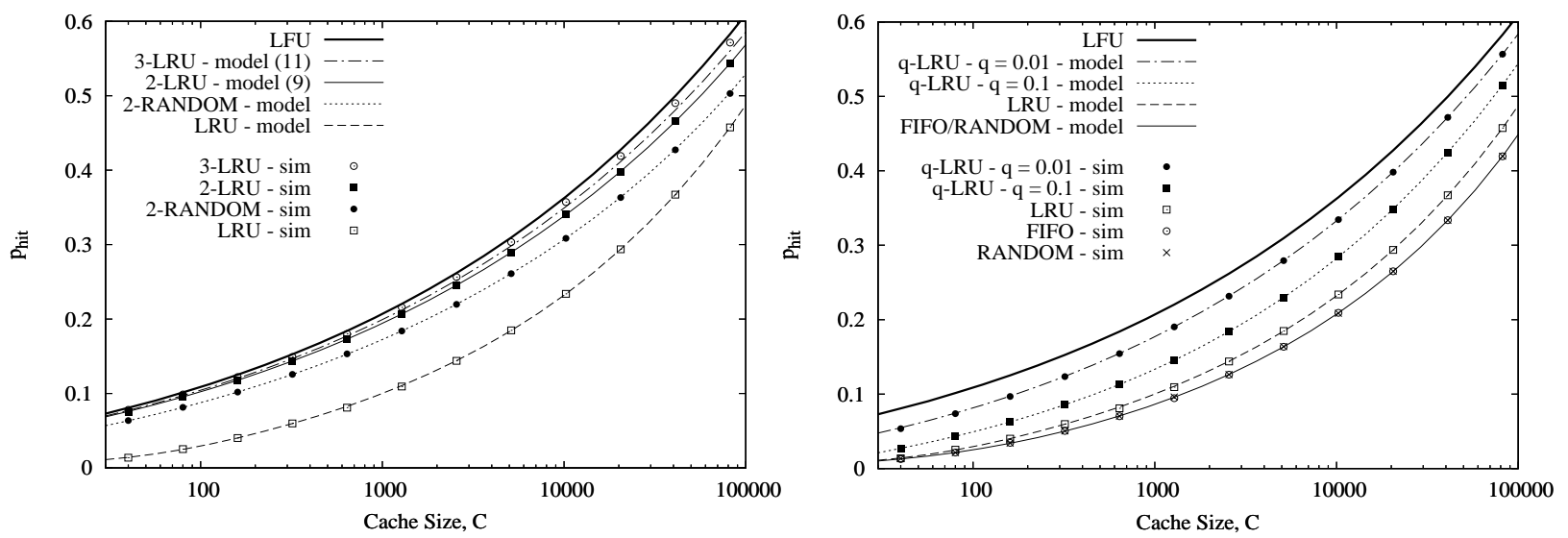

Fig. 5. Hit probability vs cache size, for various caching policies, under IRM.

\section{G. Small cache approximations}

Small cache approximations can be obtained by replacing the expressions of $p_{\text {hit }}(m)$ and $p_{\text {in }}(m)$ with their truncated Taylor expansion (with respect to $T_{C} \rightarrow 0$ ). This is especially useful to understand the dependency of $p_{\text {in }}$ and $p_{\text {hit }}$ on the object arrival rate $\lambda_{m}$ (and thus its popularity), obtaining interesting insights into the performance of the various caching policies. We restrict ourselves to IRM traffic, however we emphasize that a similar approach can be generalized to renewal traffic. We obtain:

$$
p_{\text {hit }}(m)=p_{\text {in }}(m) \approx \begin{cases}\lambda_{m} T_{C}-\frac{\left(\lambda_{m} T_{C}\right)^{2}}{2} & \text { LRU } \\ \lambda_{m} T_{C}-\left(\lambda_{m} T_{C}\right)^{2} & \text { RANDOM/FIFO } \\ q \lambda_{m} T_{C}+q\left(\frac{1}{2}-q\right)\left(\lambda_{m} T_{C}\right)^{2} & \text { q-LRU } \\ \left(\lambda_{m}\right)^{k} \prod_{i=1}^{k} T_{C}^{i} & \text { k-LRU }\end{cases}
$$

Previous expressions permit us immediately to rank the performance of the considered policies in the small cache regime. Specifically, better performance is achieved by caching policy under which $p_{\text {hit }}(m)$ exhibits stronger dependency on $\lambda_{m}$. Indeed, recall that (under IRM) $p_{\text {hit }}=\sum_{m} \frac{\lambda_{m}}{\Lambda} p_{\text {hit }}(m)$, while $\sum_{m} p_{\text {hit }}(m)=\sum_{m} p_{\text {in }}(m)=C$. Hence, the stronger the dependency of $p_{\text {hit }}(m)$ on $\lambda_{m}$, the more closely a policy tends to approximate the behavior of LFU (the optimal policy), which statically places in the cache the $C$ top popular contents.

Therefore, k-LRU turns out to be the best strategy, since the dependency between $p_{\text {hit }}(m)$ and content popularity $\lambda_{m}$ is polynomial of order $k \geq 2$, in contrast to other policies (including $q$-LRU for fixed $q$ ) for which $p_{\text {hit }}(m)$ depends linearly on $\lambda_{m}$. The coefficient of the quadratic term further allows us to rank policies other than k-LRU: $q$-LRU is the only policy exhibiting a positive quadratic term (for small $q$ ), which makes the dependency of $p_{\text {hit }}(m)$ on $\lambda_{m}$ slightly super-linear. At last LRU slightly outperforms RANDOM/FIFO because its negative quadratic term has a smaller coefficient.

\section{H. Model validation and insights}

The goal of this section is twofold. First, we wish to validate previously derived analytical expressions against simulations, showing the surprising accuracy of our approximate models in all considered cases. Second, we evaluate the impact of system/traffic parameters on cache performance, obtaining important insights for network design.

Unless otherwise specified, we will always consider a catalogue size of $M=10^{6}$, and a Zipf's law exponent $\alpha=0.8$.

Fig. 5] reports the hit probability achieved by the different caching strategies that we have considered, under IRM traffic. Analytical predictions are barely distinguishable from simulation results, also for the 3-LRU system, for which our approximation (11) relies on an additional independence assumption among the caches.

As theoretically predicted, q-LRU (k-LRU) approaches LFU as $q \rightarrow 0(k \rightarrow \infty)$. Interestingly, the introduction of a single meta-cache in front of an LRU cache (2-LRU) provides huge benefits, getting very close to optimal performance (LFU).

Differences among the hit probability achieved by the various caching policies become more significant in the small cache regime (spanning almost 1 order of magnitude). In this case, insertion policies providing some protection against unpopular objects largely outperform policies which do not filter any request. The impact of the eviction policy, instead, appears to be much weaker, with LRU providing moderately better performance than RANDOM/FIFO.

Fig. 6) shows the impact of temporal locality on caching performance: LRU is evaluated under renewal traffic in which object inter-arrival times are distributed according to a second order hyper-exponential with branches $\lambda_{m}^{1}=z \lambda_{m}$ and $\lambda_{m}^{2}=\lambda_{m} / z$ (hereinafter, we will call hyper- $z$ such distribution), so that increasing values of $z$ results into stronger temporal locality in the request process. We observe that temporal locality can have a dramatic (beneficial) impact on hit probability, hence it is crucial to take it into account while developing analytical models of cache performance. 


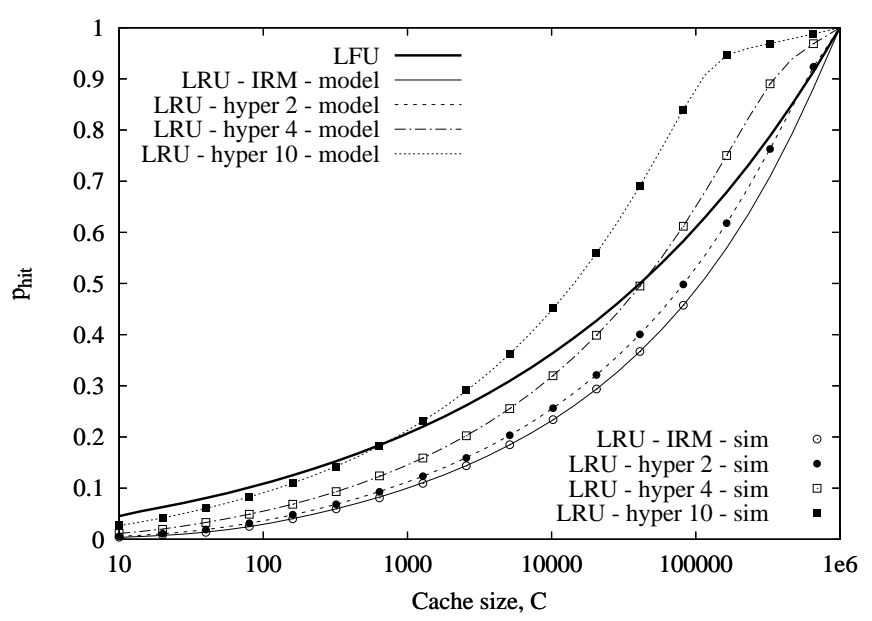

Fig. 6. Hit probability vs cache size, for LRU, under different degrees of temporal locality.
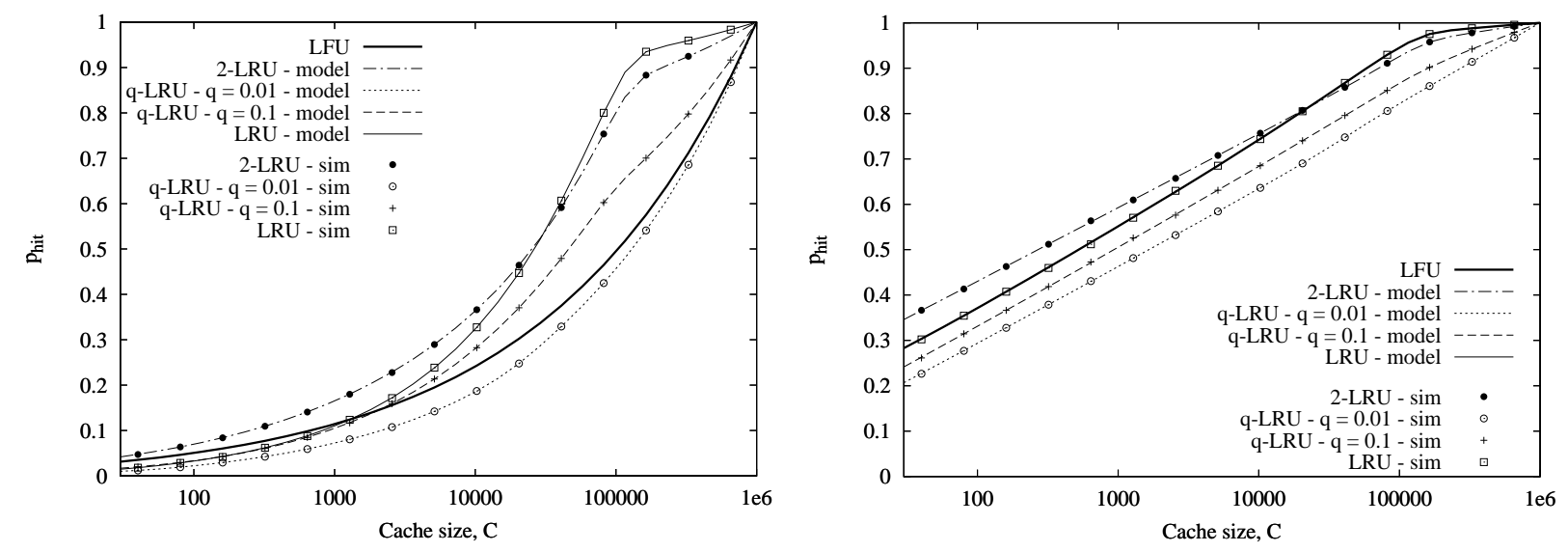

Fig. 7. Hit probability vs cache size, for various caching policies, under hyper-10 traffic, in the case of $\alpha=0.7$ (left plot) or $\alpha=1$ (right plot).

Fig. 6 also shows that LFU is no longer optimal when traffic does not satisfy the IRM. This because LFU statically places in the cache the $C$ most popular objects (on the basis of the average request rate of contents), hence the content of the cache is never adapted to instantaneous traffic conditions, resulting into suboptimal performance.

Fig. 7 compares the performance of LFU, LRU, q-LRU and 2-LRU in the case in which traffic exhibits significant temporal locality (hyper-10). We also change the Zipf's law exponent, considering either $\alpha=0.7$ (left plot) or $\alpha=1.0$ (right plot).

We observe that $q$-LRU performs poorly in this case, especially for small values of $q$ (in sharp contrast to what we have seen under IRM). This because $q$-LRU with very small $q$ tends to behave like LFU (keeping statically in the cache only the objects with the largest average arrival rate), which turns out to be suboptimal as it does not benefit from the temporal locality in the request process.

On the contrary, a simple 2-LRU system provides very good performance also in the presence of strong temporal locality. This because, while 2-LRU is able to filter out unpopular contents, its insertion policy is fast enough to locally adapt to short-term popularity variations induced by temporal locality.

To further validate the design insights gained by our analysis, we have also run a trace-driven experiment, using a real trace of Youtube video requests collected inside the network of a large Italian ISP, offering Internet access to residential customers. The trace has been extracted analysing TCP flows by means of Tstat, an open-source traffic monitoring tool developed at Politecnico di Torino [25]. During a period of 35 days in year 2012, from March 20th to April 25th, we recorded in total 3.8M of requests, for $1.76 \mathrm{M}$ of videos, coming from 31124 distinct IP addresses.

Fig. 8 reports the hit probability achieved by different caching schemes. We observe that most considerations drawn under synthetic traffic (in particular, the policy ranking) still hold when the cache is fed by real traffic taken from an operational network. We summarize the main findings: i) the insertion policy plays a crucial role in cache performance, especially in the small-cache regime; ii) a single meta-cache (2-LRU system) significantly outperforms the simple LRU and its probabilistic

\footnotetext{
${ }^{3}$ The largest cache size that we could consider was limited by the finite duration of the trace.
} 


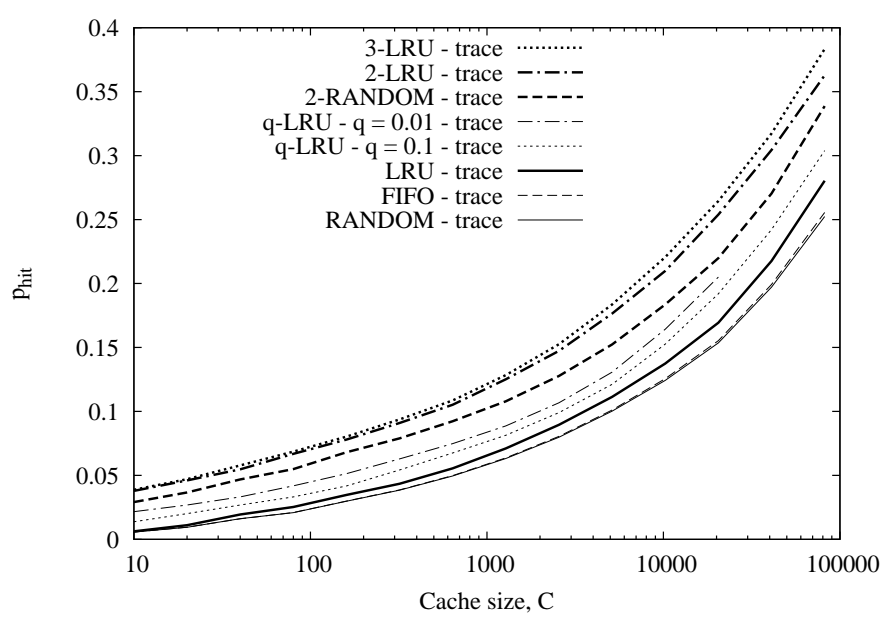

Fig. 8. Hit probability vs cache size, for various caching policies, under real trace of Youtube video requests.

version (q-LRU), while additional meta-caches provide only minor improvements; iii) the impact of the eviction policy is not significant, especially when caches are small with respect to the catalogue size.

\section{CACHE NETWORKS}

In a typical cache network, caches forward their miss stream (i.e., requests which have not found the target object) to other caches. Let us briefly recall the standard approach that has been proposed in the literature to analyse this kind of system.

We first introduce some notation. Let $p_{\text {hit }}(i, m)$ be the hit probability of object $m$ in cache $i$, and $p_{\text {in }}(i, m)$ be the (time average) probability that object $m$ is in cache $i$. We denote by $T_{C}^{i}$ the eviction time of cache $i$. Furthermore, let $\bar{\lambda}_{m}(i)$ be the total average arrival rate of requests for object $m$ at cache $i$. This rate can be immediately computed, provided that we know the hit probability of object $m$ at all caches sending their miss stream to cache $i$ - see later equation (14).

Once we know the average arrival rates $\bar{\lambda}_{m}(i)$, we can simply assume that the arrival process of requests for each object at any cache is Poisson, and thus independently solve each cache using its IRM model. A multi-variable fixed-point approach is then used to solve the entire system (see [7] for a dissection of the errors introduced by this technique).

We now explain how Che's approximation can be exploited to obtain a more accurate analysis of the cache network, under the three replication strategies defined in Sec. II-D. To describe our improved technique, it is sufficient to consider the simple case of just two caches (tandem network). Indeed, the extension of our method to general network is straightforward.

Moreover, we will limit ourselves to the case of networks of LRU caches in which the traffic produced by the users satisfies the IRM model (i.e., the exogenous process of requests for each object is Poisson). The general idea is to try to capture (though still in an approximate way) the existing correlation among the states of neighboring caches, which is totally neglected under the Poisson approximation. To do so, a different approximation is needed for each considered replication strategy, as explained in the following sections.

\section{A. Leave-copy-everywhere}

Focusing on the basic case of a tandem network, the arrival process of requests for object $m$ at the first cache is an exogenous Poisson process of rate $\lambda_{m}(1)$. The first cache (which is not influenced by the second one) can then be solved using the standard IRM model, giving

$$
p_{\text {hit }}(1, m)=p_{\text {in }}(1, m)=1-e^{-\lambda_{m}(1) T_{C}^{1}} .
$$

The arrival process of request for object $m$ at the second cache is not Poisson. It is, instead, an ON-OFF modulated Poisson process, where the ON state corresponds to the situation in which object $m$ is not stored in cache 1 , so that requests for this object are forwarded to cache 2 . Instead, no requests for object $m$ can arrive at cache 2 when $m$ is present at cache 1 (OFF state).

The standard approximation would be to compute the average arrival rate $\bar{\lambda}_{m}(2)=\lambda_{m}(1)\left(1-p_{\text {hit }}(1, m)\right)$ and to apply the IRM model also to the second cache. Can we do better than this? Actually, yes, at least to compute the hit probability $p_{\text {hit }}(2, m)$, which can, in practice, be very different from $p_{\text {in }}(2, m)$ since PASTA does not apply.

We observe that a request for $m$ can arrive at time $t$ at cache 2 , only if object $m$ is not stored in cache 1 at $t^{-}$. This implies that no exogenous requests can have arrived in the interval $\left[t-T_{C}^{1}, t\right]$ (otherwise $m$ would be present in cache 1 at time $\mathrm{t}$ ), hence, a fortiori, no requests for $m$ can have arrived at cache 2 in the same interval. 


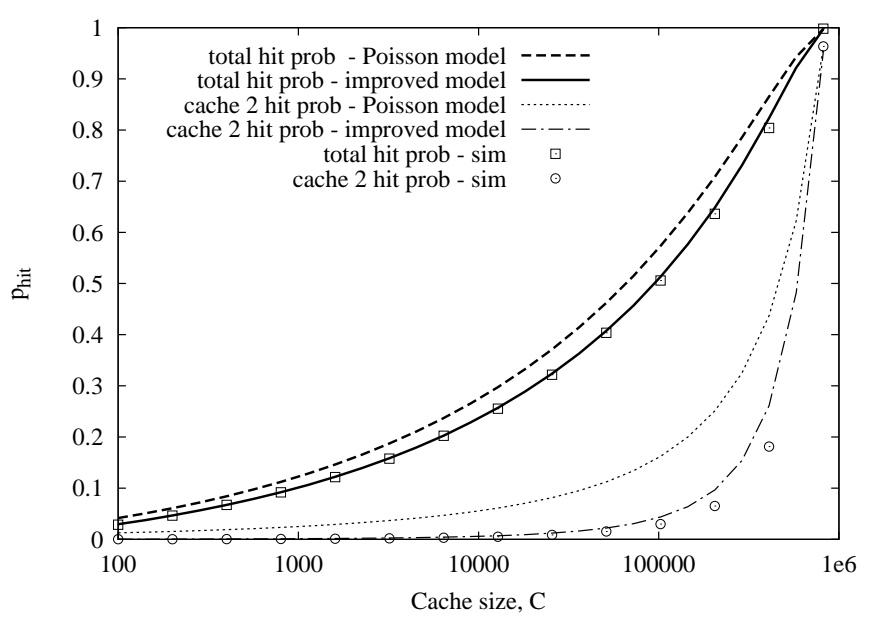

Fig. 9. Comparison between Poisson approximation and our improved approximation, in the case of a tandem network of two LRU caches, under IRM traffic

Now, provided that $T_{C}^{2}>T_{C}^{1}$, object $m$ is found in cache 2 at time $t$, if and only if at least one request arrived at cache 2 within the interval $\left[t-T_{C}^{2}, t-T_{C}^{1}\right]$. During this interval, the arrival process at cache 2 is not Poisson (it depends on the unknown state of cache 1), and we resort to approximating it by a Poisson process with rate $\bar{\lambda}_{m}(2)$, obtaining:

$$
p_{\text {hit }}(2, m) \approx 1-e^{-\bar{\lambda}_{m}(2)\left(T_{C}^{2}-T_{C}^{1}\right)}
$$

Essentially, the improvement with respect to the standard approximation consists in the term $T_{C}^{2}-T_{C}^{1}$ in the above equation, in place of $T_{C}^{2}$. If, instead, $T_{C}^{2}<T_{C}^{1}$, we clearly have $p_{\text {hit }}(2, m)=0$.

Note that the above reasoning cannot be applied to compute $p_{\text {in }}(2, m)$ (which is necessary to estimate $T_{C}^{2}$ ), thus we simply express

$$
p_{\text {in }}(2, m) \approx 1-e^{-\bar{\lambda}_{m}(2) T_{C}^{2}}
$$

as in the standard IRM model.

To show the significant gains in terms of accuracy that can be obtained by applying our simple improved approximation with respect to the Poisson approximation, we consider a tandem network in which the first cache is fed by IRM traffic with catalogue size $M=10^{6}$ and Zipf's law exponent $\alpha=0.8$. Figure 9 reports both the total hit probability and the hit probability on the second cache, under the two considered approximations, against simulation results. We observe that the Poisson approximation tends to overestimate the total hit probability, essentially as a consequence of a large overestimate of the hit probability on the second cache. Our improved approximation, which, recall, essentially leads to substituting $T_{C}^{2}$ with $T_{C}^{2}-T_{C}^{1}$ in the standard formula to compute the hit probability of the second cache, brings back the analytical prediction of total hit probability very close to simulation results, thanks to a much better model of the behavior of the second cache.

\section{B. Leave-copy-probabilistically}

Also in this case the first cache is not influenced by the second, hence we can use the IRM formula of q-LRU (5) to analyze its behavior.

To evaluate $p_{\text {hit }}(2, m)$, we observe that a request for content $m$ that arrives at time $t$ at cache 2 produces a hit if, and only if, at time $t^{-}$content $m$ is stored at cache 2 but not in cache 1 . For this to happen, in the case $T_{C}^{2}>T_{C}^{1}$ there are two sufficient and necessary conditions related to the previous request for $m$ arriving at cache 2: i) this request produced a hit at cache 2, or it triggered an insertion here; ii) it arrived at cache 2 either in the interval $\left[t-T_{C}^{2}, t-T_{C}^{1}\right]$, or in the interval $\left[t-T_{C}^{1}, t\right]$ without triggering an insertion in cache 1 . We remark that, in contrast to the LCE case, now it is possible that the previous request arrived in the interval $\left[t-T_{C}^{1}, t\right]$ : indeed, the previous request can arrive in this interval, produce a miss in cache 1 (and thus be forwarded to cache 2) and not trigger an insertion in cache 1 , so that we can really observe another request arriving at cache 2 at time $t$. To evaluate the probability of this event, we model the stream of requests arriving at cache 2 (i.e. producing a miss at cache 1) without triggering an insertion in cache 1 as a Poisson process with intensity $\bar{\lambda}_{m}(2) \cdot(1-q)$. We obtain:

$$
p_{\text {hit }}(2, m) \approx\left[p_{\text {hit }}(2, m)+q\left(1-p_{\text {hit }}(2, m)\right)\right] \cdot\left(1-e^{-\bar{\lambda}_{m}(2)\left(T_{C}^{2}-T_{C}^{1}\right)} \cdot e^{-\bar{\lambda}_{m}(2)(1-q) T_{C}^{1}}\right) .
$$

In the above expression, the first term of the product refers to condition i), whereas the second term account for condition ii) going through the complementary event that no requests arrive at cache 2 either in the interval $\left[t-T_{C}^{1}, t\right]$ nor in the interval $\left[t-T_{C}^{2}, t-T_{C}^{1}\right]$. Note that this expression reduces to (13) when $q=1$ (i.e., LCE). 
If, instead, $T_{C}^{2}<T_{C}^{1}$, the formula simplifies to

$$
p_{\text {hit }}(2, m) \approx\left[p_{\text {hit }}(2, m)+q\left(1-p_{\text {hit }}(2, m)\right)\right]\left(1-e^{-\bar{\lambda}_{m}(2)(1-q) T_{C}^{2}}\right) .
$$

To estimate $p_{\text {in }}(2, m)$, we resort to the standard Poisson approximation:

$$
p_{\text {in }}(2, m) \approx\left(1-e^{-\bar{\lambda}_{m}(2) T_{C}^{2}}\right)\left[p_{\text {in }}(2, m)+q\left(1-p_{\text {in }}(2, m)\right)\right] .
$$

\section{Leave-copy-down}

This strategy is more complex to analyse, since now the dynamics of cache 1 and cache 2 depend mutually on each other. Indeed, it is possible to insert a content in cache 1 only when it is already stored in cache 2 . Probability $p_{\text {in }}(1, m)$ can be computed considering that object $m$ is found in cache 1 if, and only if, the last request arrived in $\left[t-T_{C}^{1}, t\right]$ and either i) it hit the object in cache 1 or ii) it found the object in cache 2 (and not in cache 1). Since PASTA holds, we have:

$$
p_{\text {in }}(1, m) \approx p_{\text {hit }}(1, m)=\left[\left(1-p_{\text {in }}(1, m)\right) p_{\text {hit }}(2, m)+p_{\text {in }}(1, m)\right] \cdot\left(1-e^{-\lambda_{m}(1) T_{C}(1)}\right)
$$

Observe in the previous expression that we have assumed the states of cache 1 and cache 2 to be independent; on the other hand, similarly to what we have done before, we write:

$$
p_{\text {in }}(2, m) \approx\left(1-e^{-\bar{\lambda}_{m}(2) T_{C}^{2}}\right)
$$

Note that, since $p_{\text {in }}(1, m)$ and $p_{\text {in }}(2, m)$ are interdependent, a fixed-point iterative procedure is needed to jointly determine them.

It remains to approximate the hit probability at cache 2 . When $T_{C}^{2}>T_{C}^{1}$, we write:

$$
p_{\text {hit }}(2, m) \approx\left(1-e^{-\bar{\lambda}_{m}(2)\left(T_{C}^{2}-T_{C}^{1}\right)}\right) e^{-\bar{\lambda}_{m}(2) T_{C}^{1}}+\left(1-e^{-\bar{\lambda}_{m}(2)\left(1-p_{\text {hit }}(2, m)\right) T_{C}^{1}}\right)
$$

Indeed, since at time $t^{-}$cache 1 does not store the object by construction, either the previous request arrived in $\left[t-T_{C}^{2}, t-T_{C}^{1}\right]$ at cache 2, or it arrived in $\left[t-T_{C}^{1}, t\right]$ (again at cache 2) but it did not trigger an insertion in cache 1 because object $m$ was not found in cache 2 . As before, we model the stream of requests arriving at cache 2 (i.e. producing a miss at cache 1) without triggerring an insertion in the first cache as a Poisson process with intensity $\bar{\lambda}_{m}(2) \cdot\left(1-p_{\text {hit }}(2, m)\right)$.

Similarly, if $T_{C}^{2}<T_{C}^{1}$ :

$$
p_{\text {hit }}(2, m) \approx\left(1-e^{-\lambda_{m}(2)\left(1-p_{\text {hit }}(2, m)\right) T_{C}^{2}}\right)
$$

\section{Extension to general cache networks}

Our approach, which has been described above for the simple case of a tandem network, can be easily generalized to any network. We limit ourselves to explaining how this can be done for the leave-copy-everywhere scheme. Let $r_{j, i}$ be the fraction of requests for object $m$ which are forwarded from cache $j$ to cache $i$ (in the case of a miss in cache $j$ ). Observe that $\left[r_{j, i}\right]$ depends on the routing strategy of requests adopted in the network and can be considered as a given input to the model.

The average arrival rate of requests for $m$ at $i$ is then

$$
\bar{\lambda}_{m}(i)=\sum_{j} \bar{\lambda}_{m}(j)\left(1-p_{\text {hit }}(j, m)\right) r_{j, i}
$$

and we can immediately express:

$$
p_{\text {in }}(i, m) \approx 1-e^{-\bar{\lambda}_{m}(i) T_{C}^{i}}
$$

resorting to the standard Poisson approximation.

Our refined approach to estimating the hit probability can still be applied to the computation of the conditional probability $p_{\text {hit }}(i, m \mid j)$, which is the probability that a request for object $m$ hits the object at cache $i$, given that it has been forwarded by cache $j$. This event occurs if, and only if, either a request arrived at $i$ from $j$ in the time interval $\left[t-T_{C}^{i}, t-T_{C}^{j}\right]$ (provided that $T_{C}^{i}>T_{C}^{j}$ ), or at least one request arrived at $i$ in the interval $\left[t-T_{C}^{i}, t\right]$ from another cache (different from $j$ ). Thus we write:

$$
p_{\text {hit }}(i, m \mid j) \approx 1-e^{-A_{i, j}}
$$

where

$$
A_{i, j}=r_{j, i} \bar{\lambda}_{m}(j)\left(1-p_{i n}(j, m)\right) \max \left(0, T_{C}^{i}-T_{C}^{j}\right)+\sum_{k \neq j} r_{k, i} \bar{\lambda}_{m}(k)\left(1-p_{i n}(k, m)\right) T_{C}^{i}
$$

The expression for $p_{\text {hit }}(i, m)$ can then be obtained de-conditioning with respect to $j$.

Now, in case of tree-like networks previous expressions can be evaluated step-by-step starting from the leaves and going up towards the root. In case of general mesh networks, a global (multi-variate) fixed-point procedure is necessary. 

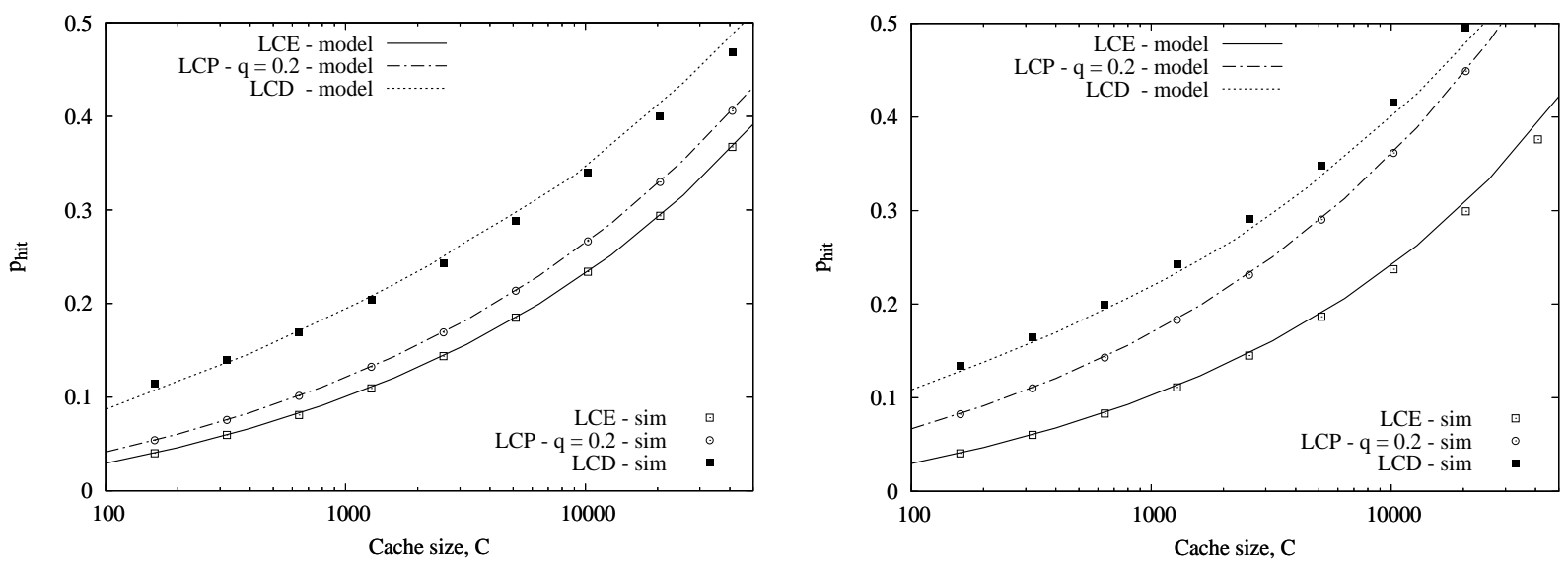

Fig. 10. Hit probability vs cache size, for various replication strategies, in the case of a chain of 6 caches, under IRM traffic. Hit probability of the first cache (left plot) and total hit probability of the network (right plot).

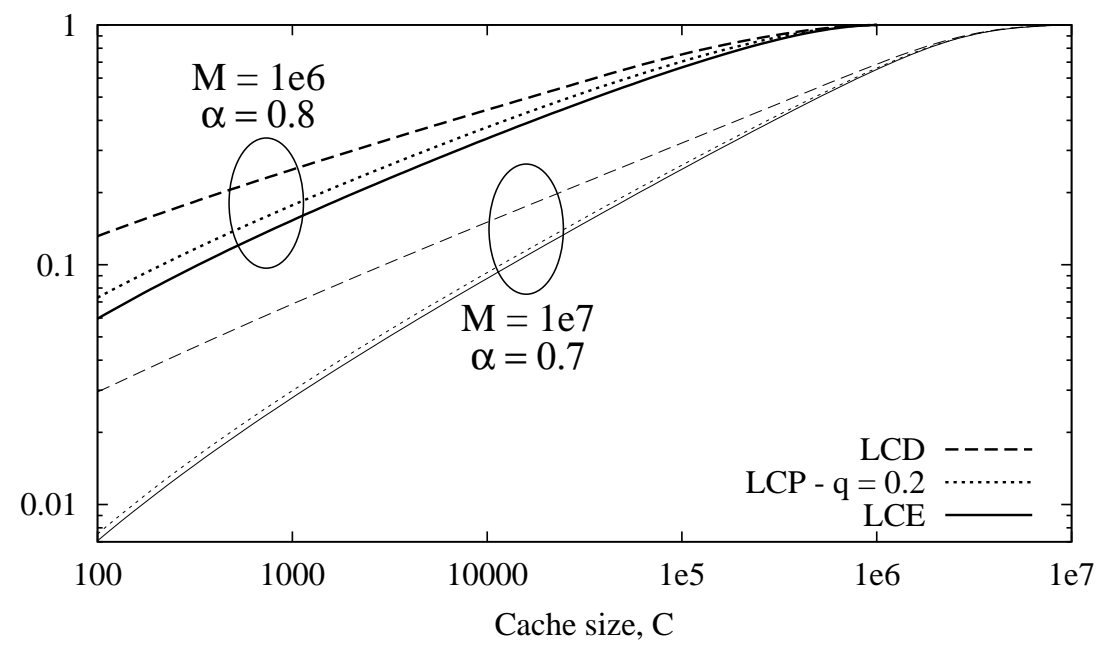

Fig. 11. Hit probability vs cache size, for various replication strategies, in the case of a tree topology with 1365 caches, for two traffic scenarios

\section{E. Model validation and insights}

As before, our aim here is to jointly validate our analytical models against simulation, while getting interesting insights into system behavior.

Fig 10 compares the performance of the different replication strategies that we have analysed, in the case of a chain of 6 identical caches. We have chosen a chain topology to validate our model, because this topology is known to produce the largest degree of correlation among caches (and thus the maximum deviation from the Poisson approximation).

We separately show the hit probability on the first cache (left plot) and the hit probability of the entire cache network (right plot), observing excellent agreement between analysis and simulation in all cases. We note that LCP significantly outperforms LCE, as it better exploits the aggregate storage capacity in the network avoiding the simultaneous placement of the object in all caches. Yet, LCD replication strategy performs even better, thanks to an improved filtering effect (LCD can be regarded as the dual of k-LRU for cache networks).

Then, we consider a very large topology comprising 1365 caches, corresponding to a 4-ary regular tree with 6 levels. Such topology is extremely expensive (if not impossible) to simulate, whereas the model can predict its behavior at the same computation cost of previous chain topology. Fig. 11 reports the total hit probability achieved in this large network, for two traffic scenarios (analytical results only).

We again observe the huge gain of LCD with respect to LCE, whereas the benefits of LCP are not very significant, especially with $\alpha=0.7$.

At last, we consider an example of mesh network comprising 9 caches arranged on a ring topology. Requests can enter the ring at any point, i.e., any of the caches along the ring acts as an ingress cache. Requests are forwarded clockwise along the ring. However, requests that have traversed 4 caches without hitting the content are redirected to a remote, common repository storing all contents. Fig. 12 shows the path followed by the requests arriving externally at one particular cache 


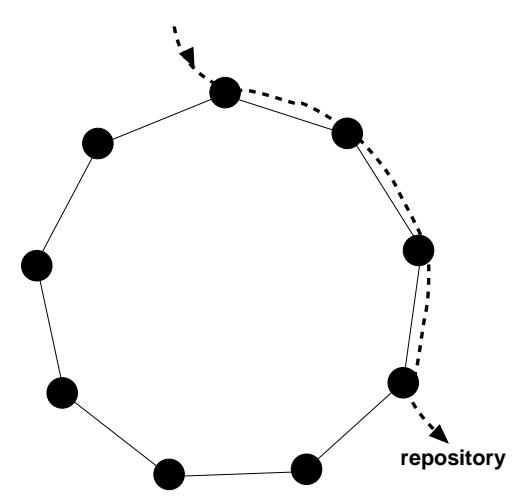

Fig. 12. Ring topology of 9 caches. The path followed by requests entering one particular cache is shown as a dashed line.

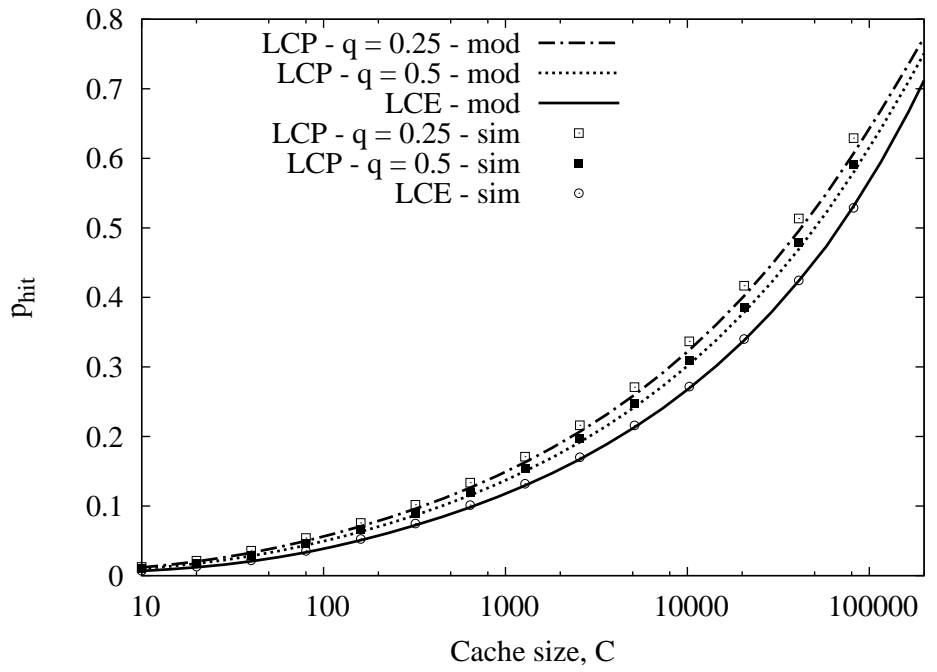

Fig. 13. Performance of LCE and LCP (with $q=0.5$ or $q=0.25$ ) in the ring topology. Comparison between analysis and simulation.

(requests entering the network at the other caches are treated in a similar way). The total external traffic of incoming requests is uniformly distributed over the 9 caches.

Fig. 13 compares the performance of LCE and LCP (with either $q=0.5$ or $q=0.25$ ) in the considered mesh network, showing the global hit probability achieved by the caching system. Here we have chosen the usual setting of $M=10^{6}$ and $\alpha=0.8$. We have not considered in this scenario the LCD replication strategy, which is primarily meant for hierarchical (tree-like) caching systems and whose performance on general networks with cyclic topology are typically worse than LCP [22].

Observe that also in the more challenging case of cache networks including cycles, the application of our model leads to pretty accurate predictions of the hit-probability. We wish to recall that networks which do not have feed-forward topology cannot be analyzed with existing techniques, such as that proposed in [26].

\section{RELATED WORK}

The literature on caching systems is vast, so we limit ourselves to mentioning the papers more closely related to our work, mainly with a modeling flavour. The first attempts to characterize the performance of simple caching systems date back to the early 70's [3], [24]. In [3] authors have shown that the computational complexity of an exact model of a single LRU or FIFO cache grows exponentially with both the cache size $C$ and the catalogue size $M$. In [24] it was proven that FIFO and RANDOM replacement policies achieve exactly the same hit probability under IRM traffic. Given that an exact characterization of most caching policies is prohibitive, approximated methodologies for the analysis of these systems have been proposed over the years [4], [5]. The work [4] proposes an approximate technique with complexity $O(C M)$ for the estimation of the hit probability in a LRU cache under IRM. The above technique can be extended also to FIFO caches, although in this case the asymptotic complexity cannot be precisely determined due to the iterative nature of the model solution. A different approximation for LRU caches under IRM was originally proposed by [5]. This approximation constitutes the starting point of our work and it is explained in detail in Sec. III

Another thread of works by Jelenković [27], [28], [29], [30] has focused on the asymptotic characterization of the hit probability in LRU caches when the catalog size and the cache size jointly scale to infinite. In particular, [27] provides a closed form expression for the asymptotic hit probability in a large LRU cache under IRM traffic with Zipf's exponent $\alpha>1$. Later works [28], [29] have shown that LRU, in the asymptotic regime, exhibits an insensitivity property to traffic temporal locality. At last [30] established the precise conditions on the scaling of parameters under which the insensitivity property holds. More recently, in [21], the same author proposed the "persistent-access-caching" (PAC) scheme, showing that it provides nearly-optimal asymptotic performance under IRM with Zipf's exponent $\alpha>1$. We emphasize that the idea behind the PAC scheme shares some similarities with the $k$-LRU scheme proposed in this work: under both schemes an insertion policy is added to LRU to prevent unpopular contents from entering the cache. However, the configuration of PAC is harder as it requires setting several parameters. The $k$-LRU scheme, instead, is simpler and self-adapting. Other generalizations/extensions of LRU known as LRU-2Q, LRU- $k$ and LRFU have been proposed in [20], [31] and [32] respectively. LRU-2Q is essentially equivalent to $k$-LRU, in the case of $k=2$. Both LRU- $k$ and LRFU, instead, subsume either LRU or LFU by making the choice of the content to be evicted dependent by the pattern of last $k$ observed content-requests. $k$-LRU is somehow complementary to both 
LRU- $k$ and LRFU, since it enhances only the insertion policy of the classical LRU, by restricting access to the cache only to those contents which are sufficiently popular, while preserving the simplicity of LRU eviction.

In the last few years cache systems have attracted renewed interest in the context of ICN. In [33] a Markovian approach has been proposed to approximate the hit probability in LRU caches under IRM. The proposed method, however, is based on Markovian assumptions and cannot be easily extended to non-IRM traffic. In [34] the approach of [27] has been extended to analyze the chunkization effect on cache performance in an ICN context. An asymptotic characterization (for large caches) of the hit probability achieved by the RANDOM policy is provided in [8]. The trade-off between recency and frequency in LRFU has been studied in [35].

The work [6] provides a theoretical justification to Che's approximation for LRU, and introduces a first attempt to apply Che's approach to non-LRU caches, considering the RANDOM policy under IRM. We emphasize that the approach proposed in [6] to analyse RANDOM differs substantially from ours, being significantly more complex and hardly extendible to non-IRM traffic. At last, we wish to mention that Che's approximation for LRU has been very recently [9] extended to non-IRM traffic in special cases, adopting a dual approach with respect to ours.

With respect to all of the above-mentioned works, the goal of our paper is different, in that here we show that the decoupling principle underlying Che's approximation is much more general and flexible than what originally thought, and can be successfully applied to a broad set of caching policies under different traffic conditions, within a unified framework.

For what concerns cache networks, we mention [36], [7], [8]. The work [36] explores ergodicity conditions for arbitrary (mesh) networks. The models in [7] and [8] rely on the independence assumption among caches, assuming that requests arriving at each cache satisfy the IRM assumptions. In contrast, we propose a methodology to capture the existing correlation among the states of neighboring caches, in a computationally efficient manner, considerably improving the accuracy of analytical predictions. Our approach also permits analyzing cache networks adopting tightly coordinated replication strategies such as leave-copy-down. We remark that cache networks implementing LCD have been previously considered in [37] for the special case of tandem topologies. Our methodology provides a significantly simpler and higher scalable alternative to the approach devised in [37], by capturing in a simple yet effective way existing correlations between caches' states, while reducing the number of parameters that must be estimated through fixed-point procedure.

Finally an alternative approach to ours has been recently proposed in [26],[38],[39] for cache networks with feed-forward topology, implementing TTL-based eviction policies. Their approach, which can be used to analyse the performance of LRU, RANDOM and FIFO under the Che approximation, essentially consists in characterizing the inter-request process arriving at non-ingress caches through a two steps procedure: i) the miss stream of (ingress) caches is exactly characterized as a renewal process with given distribution; ii) by exploiting known results on the superposition of independent renewal processes, the exact inter-request time distribution at non-ingress caches is obtained. Observe, however, that the request process at non-ingress caches are, in general, non-renewal (since the superposition of independent renewal processes is not guaranteed to be renewal). Thus, while the procedure proposed in [26] is exact for network of TTL caches with linear topology, it can be applied to network of caches with tree-structure only by approximating the request processes at non-ingress caches with renewal processes. Recently a nice refinement of the approach followed by [26] has been proposed in [40], where it has shown that the miss stream of TTL-based caches is a Markovian arrival process (MAP), provided that the request process is MAP. In light of the fact that the superposition of independent MAPs is also a MAP, [40] has derived an exact analytical method for the analysis of feed-forward networks of TTL caches under MAP traffic.

Although the approach in [26] and [40] is very elegant, and can be potentially extended to renewal traffic, it suffers from the following two limitations: i) it becomes computationally very intensive when applied to large networks; ii) it can be hardly generalized to general mesh networks (non feed-forward). Our approach is somehow complementary to the one followed by [26] and [40] since, while it applies only to IRM traffic, it is much more scalable and readily applicable to networks with general topology.

\section{CONCLUSIONS}

The main goal of this paper was to show that a variety of caching systems (both isolated and interconnected caches) operating under various insertion/eviction policies and traffic conditions, can be accurately analysed within a unified framework based on a fairly general decoupling principle extending the original Che's approximation. We have also shown that many properties of cache systems can be obtained within our framework in a simple and elegant way, including asymptotic results which would otherwise require significant efforts to be established. From the point of view of system design, our study has revealed the superiority of the k-LRU policy, in terms of both simplicity and performance gains. Still many extensions and refinements are possible, especially for cache networks under general traffic. 


\section{APPENDIX}

\section{APPENDIX}

We first prove that $\lim _{q \rightarrow 0} T_{C}=+\infty$. Consider function $f\left(T_{C}, q\right) \triangleq \sum_{m} p_{\text {in }}(m)$, From (2), $f\left(T_{C}, q\right) \equiv C$. Recalling (5), we have:

$$
f\left(T_{C}, q\right)=\sum_{m} \frac{q\left(1-e^{-\lambda_{m} T_{C}}\right)}{e^{-\lambda_{m} T_{C}}+q\left(1-e^{-\lambda_{m} T_{C}}\right)}
$$

where previous sum extends over all contents in the catalog (which is assumed to be of finite size $M$ ). Deriving the above formula, we obtain:

$$
f_{q} \triangleq \frac{\partial f}{\partial q}=\left.\sum_{m} \frac{(1-x)(x+q(1-x))-q(1-x)^{2}}{(x+q(1-x))^{2}}\right|_{x=e^{-\lambda_{m} T_{C}}}=\left.\sum_{m} \frac{(1-x) x}{(x+q(1-x))^{2}}\right|_{x=e^{-\lambda_{m} T_{C}}}>0
$$

and

$$
\begin{aligned}
& f_{T_{C}} \triangleq \frac{\partial f}{\partial T_{C}}=\left.\frac{\partial f}{\partial x}\right|_{x=e^{-\lambda_{m} T_{C}}} \frac{\partial e^{-\lambda_{m} T_{C}}}{\partial T_{C}}=\sum_{m} \partial\left(\frac{q(1-x)}{x+q(1-x)}\right) /\left.\partial x\right|_{x=e^{-\lambda_{m} T_{C}}}\left(-\lambda_{m} e^{-\lambda_{m} T_{C}}\right)= \\
&=\left.\sum_{m} \frac{-q(x+q(1-x))-q(1-x)(1-q)}{(x+q(1-x))^{2}}\right|_{x=e^{-\lambda_{m} T_{C}}}\left(-\lambda_{m} e^{-\lambda_{m} T_{C}}\right)= \\
&=\sum_{m} \frac{q \lambda_{m} e^{-\lambda_{m} T_{C}}}{\left(e^{-\lambda_{m} T_{C}}+q\left(1-e^{-\lambda_{m} T_{C}}\right)\right)^{2}}>0
\end{aligned}
$$

By the implicit function theorem, we have

$$
\frac{\partial T_{C}}{\partial q}=\frac{-f_{q}\left(T_{C}, q\right)}{f_{T_{C}}\left(T_{C}, q\right)}<0
$$

We can conclude that $T_{C}$ is a decreasing function with respect to $q$, thus we have that the $\operatorname{limit}_{\lim _{q \rightarrow 0}} T_{C}$ exists and equals $\sup _{q} T_{C}$. We prove now that this limit is indeed equal to infinity. We define $T_{C \text {,sup }} \triangleq \sup _{q} T_{C}(q)=\lim _{q \rightarrow 0} T_{C}$, and we suppose by contradiction that this is a finite quantity. In this case, we would have

$$
\lim _{q \rightarrow 0} f\left(T_{C}, q\right)=\lim _{q \rightarrow 0} \sum_{m} p_{\text {in }}(m)=\lim _{q \rightarrow 0} \sum_{m} \frac{q\left(1-e^{-\lambda_{m} T_{C}}\right)}{e^{-\lambda_{m} T_{C}}+q\left(1-e^{-\lambda_{m} T_{C}}\right)}=0,
$$

in contrast with the fact that the previous sum is equal to $C$, by definition. Thus, $T_{C, \text { sup }} \triangleq \lim _{q \rightarrow 0} T_{C}(q)=+\infty$. We prove now that $T_{C}(q)$ asymptotically behaves as $c \log \frac{1}{q}$ for some $c>0$ as $q \rightarrow 0$. We can write:

$$
\begin{aligned}
& \lim _{q \rightarrow 0} \sum_{m} p_{\text {in }}(m)= \\
& \lim _{q \rightarrow 0} \sum_{m} \frac{\left.q\left(1-e^{-\lambda_{m} T_{C}}\right)\right)}{e^{-\lambda_{m} T_{C}}+q\left(1-e^{-\lambda_{m} T_{C}}\right)}= \\
& \lim _{q \rightarrow 0} \sum_{m} \frac{q+o(q)}{e^{-\lambda_{m} T_{C}}+q+o(q)}= \\
& \lim _{q \rightarrow 0} \sum_{m} \frac{1+o(1)}{1+e^{-\lambda_{m} T_{C}} / q+o(1)}= \\
& \lim _{q \rightarrow 0} \sum_{m} \frac{1+o(1)}{1+e^{-\left(\lambda_{m} T_{C}-\log (1 / q)\right)}+o(1)}
\end{aligned}
$$

We note that, if $\frac{T_{C}}{\log (1 / q))}$ becomes arbitrarily large as $q \rightarrow 0$, every term in 177 tends to 1 , and the sum would be equal to the number of contents, whereas we know that it has to be equal to $C$. If, on the other hand, $\frac{T_{C}}{\log (1 / q))}$ becomes arbitrarily small as $q \rightarrow 0$, every term in the sum in 177 would tend to 0 . We can thus conclude that $\frac{T_{C}}{\log (1 / q)}$ is bounded away from both 0 and infinite.

Thus assuming for the moment that $\lim _{q \rightarrow 0} \frac{T_{C}}{\log (1 / q))}$ exists, it must necessarily be equal to $c>0$. Now, by setting $\lambda^{*}=1 / c$ we have:

$$
\lim _{q \rightarrow 0} p_{\text {in }}(m)=\lim _{q \rightarrow 0} \frac{1+o(1)}{q^{\frac{\lambda_{m}}{\lambda^{*}}-1}+1+o(1)}= \begin{cases}1 & \text { if } \lambda_{m} \geq \lambda^{*} \\ 0 & \text { if } \lambda_{m}<\lambda^{*}\end{cases}
$$

Note that previous argument still holds when $\lim _{q \rightarrow 0} \frac{T_{C}}{\log (1 / q))}$ does not exist, provided that the following condition is met: i) no $\lambda_{m}$ can be found, with $\lambda^{*}<\lambda_{m} \leq \Lambda^{*}$, such that $0<\liminf _{q \rightarrow 0} \frac{T_{C}}{\log (1 / q))}=\frac{1}{\Lambda^{*}}<\lim \sup _{q \rightarrow 0} \frac{T_{C}}{\log (1 / q))}=\frac{1}{\lambda_{m}}<\infty$.

At last we show, by contradiction, that either $\lim _{q \rightarrow 0} \frac{T_{C}}{\log (1 / q))}$ exists or condition i) above is met. Indeed, assume that there is an $m$ such that $\lambda^{*} \leq \lambda_{m}<\Lambda^{*}$. Then, denoting with $\mathbb{I}_{\{A\}}$ the indicator function associated to the event $\{A\}$, by construction it must be both $\sum_{m} \mathbb{I}_{\left\{\lambda_{m} \geq \lambda^{*}\right\}}=C$ and $\sum_{m} \mathbb{I}_{\left\{\lambda_{m} \geq \Lambda^{*}\right\}}=C$. Thus: $\sum_{m} \mathbb{I}_{\left\{\lambda_{m} \geq \lambda^{*}\right\}}=\sum_{m} \mathbb{I}_{\left\{\lambda_{m} \geq \Lambda^{*}\right\}}$, which is in contradiction 
with the assumption.

To simplify the proof we assume the support of the inter-request time pdf to be simply connected. As consequence, $F(m, y)$ $(\hat{F}(m, y))$ is a strictly increasing function with respect to variable $x(y)$ on its relevant range, i.e, for any $x$ such that $0<$ $F(m, x)<1(\forall y$ s.t. $0<\hat{F}(m, y)<1)$. First we consider to the case in which $F(m, x)(\hat{F}(m, y))$ has an infinite support for any $m$. In this case we first prove that $\lim _{q \rightarrow 0} T_{C}=+\infty$. Consider function $f\left(T_{C}, q\right) \triangleq \sum_{m} p_{\text {in }}(m)$. From (2), $f\left(T_{C}, q\right) \equiv C$. Recalling (7) and (6), we have:

$$
p_{\text {hit }}(m)=\frac{q F\left(m, T_{C}\right)}{1-F\left(m, T_{C}\right)(1-q)}
$$

and

$$
\begin{aligned}
& p_{\text {in }}(m)=\hat{F}\left(m, T_{C}\right)\left[p_{\text {hit }}(m)+q\left(1-p_{\text {hit }}(m)\right)\right]= \\
& \quad \hat{F}\left(m, T_{C}\right)\left[\frac{q F\left(m, T_{C}\right)}{1-F\left(m, T_{C}\right)(1-q)}+q\left(1-\frac{q F\left(m, T_{C}\right)}{1-F\left(m, T_{C}\right)(1-q)}\right)\right]=\hat{F}\left(m, T_{C}\right) \frac{q}{1-F\left(m, T_{C}\right)(1-q)}
\end{aligned}
$$

Thus,

$$
f\left(T_{C}, q\right)=\sum_{m} \hat{F}\left(m, T_{C}\right) \frac{q}{1-F\left(m, T_{C}\right)(1-q)} .
$$

Deriving this formula, we obtain:

$$
f_{q} \triangleq \frac{\partial f}{\partial q}=\sum_{m} \hat{F}\left(m, T_{C}\right) \frac{1-F\left(m, T_{C}\right)(1-q)-q F\left(m, T_{C}\right)}{\left[1-F\left(m, T_{C}\right)(1-q)\right]^{2}}=\sum_{m} \hat{F}\left(m, T_{C}\right) \frac{1-F\left(m, T_{C}\right)}{\left[1-F\left(m, T_{C}\right)(1-q)\right]^{2}}>0
$$

and

$$
\begin{aligned}
f_{T_{C}} \triangleq \frac{\partial f}{\partial T_{C}}=\sum_{m} \frac{\partial \hat{F}\left(m, T_{C}\right)}{\partial T_{C}}\left[\frac{q}{1-F\left(m, T_{C}\right)(1-q)}\right]+\hat{F}\left(m, T_{C}\right) \frac{\partial}{\partial T_{C}} \frac{q}{1-F\left(m, T_{C}\right)(1-q)}= \\
=\sum_{m} \frac{\partial \hat{F}\left(m, T_{C}\right)}{\partial T_{C}}\left[\frac{q}{1-F\left(m, T_{C}\right)(1-q)}\right]+\hat{F}\left(m, T_{C}\right) \frac{q(1-q)}{\left[1-F\left(m, T_{C}\right)(1-q)\right]^{2}} \frac{\partial F\left(m, T_{C}\right)}{\partial T_{C}}>0
\end{aligned}
$$

since both $\hat{F}\left(m, T_{C}\right)$ and $F\left(m, T_{C}\right)$ are increasing with $T_{C}$.

By the implicit function theorem, we have

$$
\frac{\partial T_{C}}{\partial q}=\frac{-f_{q}\left(T_{C}, q\right)}{f_{T_{C}}\left(T_{C}, q\right)}<0
$$

We can conclude that $T_{C}$ is a decreasing function with respect to $q$, thus we have that the $\operatorname{limit} \lim _{q \rightarrow 0} T_{C}$ exists and equals $\sup _{q} T_{C}$. We prove now that this limit is indeed equal to infinity. We define $T_{C \text {,sup }} \triangleq \sup _{q} T_{C}(q)=\lim _{q \rightarrow 0} T_{C}$, and we suppose, by contradiction, that this is a finite quantity. In this case, we would have

$$
\lim _{q \rightarrow 0} f\left(T_{C}, q\right)=\lim _{q \rightarrow 0} \sum_{m} p_{\text {in }}(m)=\lim _{q \rightarrow 0} \sum_{m} \hat{F}\left(m, T_{C}\right) \frac{q}{1-F\left(m, T_{C}\right)(1-q)}=0,
$$

in contrast with the fact that the previous sum is equal to $C$, by definition. Thus, since $\lim _{q \rightarrow 0} T_{C}(q)=+\infty$ we have:

$$
\lim _{q \rightarrow 0} p_{\text {in }}(m)=\lim _{q \rightarrow 0} \sum_{m} \hat{F}\left(m, T_{C}\right) \frac{1}{\left[1-F\left(m, T_{C}\right)(1-q)\right] / q},
$$

now, observe that if $1-F\left(m, T_{C}\right)=o(q)$, the previous limit becomes equal to 1 , whereas, if $1-F\left(m, T_{C}\right)=\omega(q)$, the limit is equal to 0 .

Then, with similar arguments as for the exponential case, under our assumptions (i.e., the fact that we assume $\lim _{t \rightarrow \infty} \frac{1-F\left(m_{1}, t\right)}{1-F\left(m_{2}, t\right)}=$ $\infty$ whenever $\lambda_{m_{1}}<\lambda_{m_{2}}$ ) we can easily show that there necessarily exists some $m_{0}$ such that $1-F\left(m, T_{C}\right)=o(q)$ for $m<m_{0}$ and $1-F\left(m, T_{C}\right)=\omega(q)$ for $m>m_{0}$. Indeed observe that, by hypothesis, the asymptotic behavior of $1-F\left(m, T_{C}\right)$ as $T_{C} \rightarrow \infty$ depends on $m$ (i.e., on arrival rates $\lambda_{m}$ 's, which are assumed to be different for different $m$ ).

Fig. 14 provides a numerical confirmation of our theoretical predictions (see also Remark after Theorem 4.1), plotting the hit probability as function of the insertion probability of q-LRU under different inter-request time distributions: exponential, hyper-10, Pareto (with exponent $\gamma=2$ ). This experiment suggests that both the exponential and hyper-10 curves approach LFU as $q \rightarrow 0$, while the curve corresponding to the Pareto case tends to a different limit.

The case in which $F\left(m, T_{C}\right)$ has a bounded support for some $m$ can be treated similarly. However if the number of contents with finite support exceeds $C, T_{C}$ does not tend anymore to $\infty$. Observe indeed that from 19 we can deduce that every

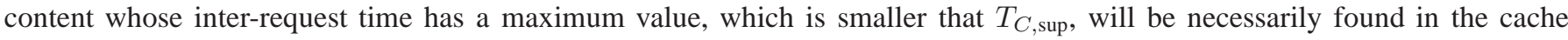
with a probability tending to 1 when $q \rightarrow 0$, while every other content will be found with a probability tending to 0 . Thus, since by construction we have $\sum_{m} P_{\text {in }}(m)=C$, only $C$ contents can have maximum inter-request time smaller than $T_{C \text {,sup. }}$. 


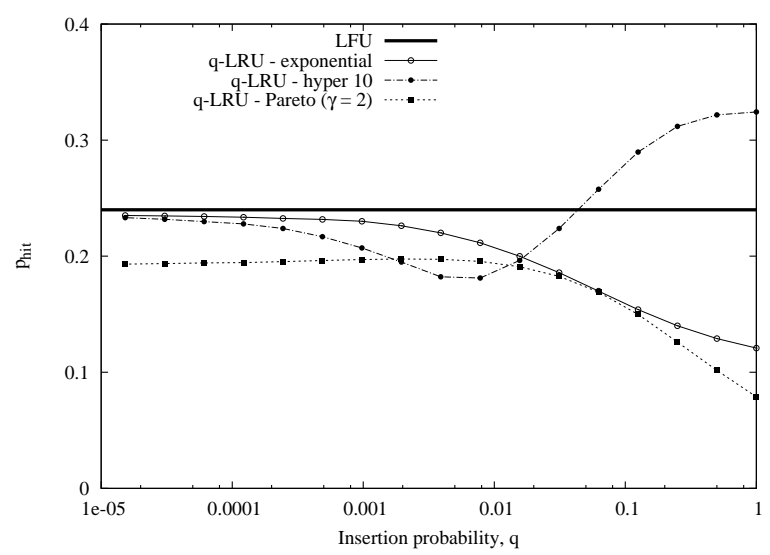

Fig. 14. Hit probability vs insertion probability of q-LRU, for different inter-request time distributions, fixed cache size equal to $10,000, \alpha=0.7$.

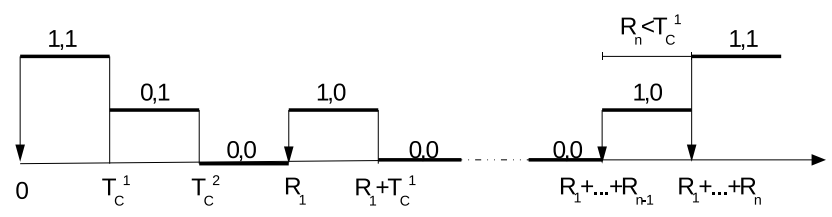

Fig. 15. Illustration of the cycle analysis used for deriving 20. Vertical arrows represent incoming requests for the content.

This concludes the proof.

For simplicity in this appendix, whenever not strictly necessary, we omit the dependency of variables on $m$. We define as cycle the time interval between two visits at state $(1,1)$ (i.e., the time interval between two requests for object $m$ that bring the system to state $(1,1))$. Observe that, by construction, the cycles are i.i.d. We consider a generic cycle starting at time $t=0$ (thus by construction a request for $m$ arrives at time $t=0$ ). Let $R_{1}$ be the time of the first request for object $m$ after $t=0$. We have the following possibilities:

- $R_{1} \leq T_{C}^{1}$ : at time $R_{1}^{-}$the system is still in state $(1,1)$, and consequently $\mathbb{E}\left[T_{\text {cycle }} \mid R_{1}<T_{C}^{1}\right]=\mathbb{E}\left[R_{1} \mid R_{1}<T_{C}^{1}\right]$.

- $T_{C}^{1}<R_{1} \leq T_{C}^{2}$ : in this case, at time $t=T_{C}^{1}$ the system enters state $(0,1)$, where it is found at $R_{1}^{-}$; thus the request at $R_{1}$ brings the system again in state $(1,1)$. In this case $\mathbb{E}\left[T_{\text {cycle }} \mid T_{C}^{1}<R_{1} \leq T_{C}^{2}\right]=\mathbb{E}\left[R_{1} \mid T_{C}^{1}<R_{1} \leq T_{C}^{2}\right]$.

- $R_{1}>T_{C}^{2}$ : in this last case the analysis is more complicated. At time $T_{C}^{1}$ the system goes to state $(0,1)$, and at time $T_{C}^{2}$ it enters state $(0,0)$. At time $t=R_{1}$, for effect of the arrival of the first request, the system enters $(1,0)$. Now, if the following request arrives before $R_{1}+T_{C}^{1}$, the system goes back to state $(1,1)$ and the cycle terminates; otherwise, the system at time $R_{1}+T_{C}^{1}$ enters again state $(0,0)$ and the following request brings it again to state $(1,0)$. The cycle ends upon the arrival of the first request for object $m$ that follows the previous one by less than $T_{C}^{1}$. Figure 15 better illustrates this situation.

Thus, if we denote by $R_{i}$ the $i$-th inter-request time, and with $n \sim \operatorname{Geom}\left(p_{1}\right), p_{1}=\mathbb{P}\left(R \leq T_{C}^{1}\right)=1-e^{-\lambda_{m} T_{C}^{1}}$ and $p_{2}=\mathbb{P}\left(R \leq T_{C}^{2}\right)=1-e^{-\lambda_{m} T_{C}^{2}}$, we can write in this case:

$$
\begin{aligned}
\mathbb{E}\left[T_{\text {cycle }} \mid R_{1}>T_{C}^{2}\right] & =\mathbb{E}\left[R_{1} \mid R_{1}>T_{C}^{2}\right]+\mathbb{E}\left[R_{n} \mid R_{n} \leq T_{C}^{1}\right]+\mathbb{E}\left[\sum_{i=0}^{n-1} R_{i} \mid R_{i}>T_{C}^{1}\right] \\
& =\mathbb{E}\left[R_{1} \mid R_{1}>T_{C}^{2}\right]+\mathbb{E}\left[R_{n} \mid R_{n} \leq T_{C}^{1}\right]+\mathbb{E}[n] \mathbb{E}\left[R_{i} \mid R_{i}>T_{C}^{1}\right] \\
& =\mathbb{E}\left[R_{1} \mid R_{1}>T_{C}^{2}\right]+\frac{\mathbb{E}\left[R_{n}, R_{n} \leq T_{C}^{1}\right]}{\mathbb{P}\left(R_{n} \leq T_{C}^{1}\right)}+\frac{1-p_{1}}{p_{1}} \frac{\mathbb{E}\left[R_{i}, R_{i}>T_{C}^{1}\right]}{\mathbb{P}\left(R_{i}>T_{C}^{1}\right)} \\
& =\mathbb{E}\left[R_{1} \mid R_{1}>T_{C}^{2}\right]+\frac{\mathbb{E}\left[R_{n}, R_{n} \leq T_{C}^{1}\right]}{p_{1}}+\frac{1-p_{1}}{p_{1}} \frac{\mathbb{E}\left[R_{i}, R_{i}>T_{C}^{1}\right]}{1-p_{1}} \\
& =\mathbb{E}\left[R \mid R>T_{C}^{2}\right]+\frac{\mathbb{E}[R]}{p_{1}}
\end{aligned}
$$


Considering also the other cases, we have:

$$
\begin{aligned}
\mathbb{E}\left[T_{\text {cycle }}\right] & =\mathbb{E}\left[R_{1} \mid R_{1}<T_{C}^{2}\right] \mathbb{P}\left(R_{1} \leq T_{C}^{2}\right)+\left(\mathbb{E}\left[R_{1} \mid R_{1}>T_{C}^{2}\right]+\frac{\mathbb{E}[R]}{p_{1}}\right) \mathbb{P}\left(R_{1}>T_{C}^{2}\right) \\
& =\mathbb{E}[R]+\frac{\mathbb{E}[R]}{p_{1}}\left(1-p_{2}\right)
\end{aligned}
$$

Turning our attention to $\mathbb{E}\left[T_{\mathrm{ON}}\right]$, which is the average time within a cycle during which content $m$ is stored in the second (physical) cache, we have:

$$
\mathbb{E}\left[T_{\mathrm{ON}}\right]=\mathbb{E}\left[\min \left(R_{1}, T_{C}^{2}\right)\right]=\mathbb{E}\left[R_{1} \mid R_{1}<T_{C}^{2}\right] \mathbb{P}\left(R_{1}<T_{C}^{2}\right)+T_{C}^{2} \mathbb{P}\left(R_{1} \geq T_{C}^{2}\right)
$$

At last we can obtain $p_{\text {in }}(m)$ as:

$$
p_{\text {in }}(m)=\frac{\mathbb{E}\left[T_{\mathrm{ON}}(m)\right]}{\mathbb{E}\left[T_{\text {cycle }}(m)\right]}
$$

For simplicity we limit ourselves to the IRM traffic model. An analogous result can be derived under renewal traffic along the same lines. First we recall that sequence $\left\{T_{C}^{i}\right\}_{i=1}^{k}$ is increasing. We prove that $T_{C}^{*}=\sup _{k \rightarrow \infty} T_{C}^{k}=+\infty$. Indeed, assume by contradiction that $T_{C}^{*}$ is finite. Now, a necessary condition for content $m$ to be in the cache at time $t$ is that a request arrived at $\tau_{1} \in\left(t-T_{C}^{k}, t\right]$; this request in turn must have necessarily generated a hit either in cache $k$ or in cache $k-1$. As consequence, a previous request must have arrived at $\tau_{2} \in\left(\tau_{1}-T_{C}^{k}, \tau_{1}\right]$. Iterating back we generate a chain of $k$ requests for object $m$ requests with inter-request time smaller than $T_{C}^{k}$, which is necessary for object $m$ to be found in cache $k$ at time $t$. The probability of observing such a chain is bounded by $\left(1-e^{-\lambda_{m} T_{C}^{*}}\right)^{k}$, and this probability goes to zero when $k \rightarrow \infty$, independently on $\lambda_{m}$, leading to a contradiction. Indeed recall that, by construction, $\sum p_{\text {in }}(m, k)=C$. Thus, we can conclude that $\lim _{k \rightarrow \infty} T_{C}=+\infty$. Recalling the expression in (11):

$$
p_{\text {in }}(m, i)=\left(1-e^{\left.-\lambda_{m} T_{C}^{i}\right)}\right)\left[p_{\text {in }}(m, i)+\left(p_{\text {in }}(m, i-1)\right)\left(1-p_{\text {in }}(m, i)\right)\right]
$$

we can easily prove that: $p_{\text {in }}(m, i)$ is increasing with respect to $\lambda_{m}$ for any $i$ (by induction over $i$ ); ii) 111, for sufficiently large $T_{C}^{i}$, is a contraction mapping over $[\epsilon, 1]$ for any $\epsilon>0$.

Thus, $\lim _{k \rightarrow \infty} p_{\text {in }}(m, k)$ exists and it is necessarily the fixed point $p_{\mathrm{in}}^{*}(m)$ of 111). The assertion immediately follows, since $p_{\text {in }}^{*}(m) \in\{0,1\}$.

The extension to the non-IRM case, under the assumption that the support of the inter-request time distribution is unbounded, and that for any $m_{1}$ and $m_{2}$, with $\lambda_{m_{1}}<\lambda_{m_{2}}, \lim _{t \rightarrow \infty} \frac{1-F\left(m_{1}, t\right)}{1-F\left(m_{2}, t\right)}>1$, follows the same lines.

\section{REFERENCES}

[1] W. Jiang, S. Ioannidis, L. Massoulié, and F. Picconi, "Orchestrating massively distributed CDNs," in ACM CoNEXT, 2012.

[2] V. Jacobson, D. K. Smetters, J. D. Thornton, M. F. Plass, N. H. Briggs, and R. L. Braynard, "Networking named content," in ACM CoNEXT, 2009.

[3] W. King, Analysis of Paging Algorithms. IBM-Report, 1971.

[4] A. Dan and D. Towsley, "An approximate analysis of the LRU and FIFO buffer replacement schemes," SIGMETRICS Perform. Eval. Rev., vol. 18, pp. 143-152, Apr. 1990.

[5] H. Che, Y. Tung, and Z. Wang, "Hierarchical Web caching systems: modeling, design and experimental results," IEEE JSAC, vol. 20, no. 7, pp. 1305-1314, 2002.

[6] C. Fricker, P. Robert, and J. Roberts, "A versatile and accurate approximation for lru cache performance," ITC, 2012.

[7] E. Rosensweig, J. Kurose, and D. Towsley, “Approximate Models for General Cache Networks," in INFOCOM, 2010.

[8] M. Gallo, B. Kauffmann, L. Muscariello, A. Simonian, and C. Tanguy, "Performance evaluation of the random replacement policy for networks of caches," SIGMETRICS Perf. Eval. Rev., vol. 40(1), pp. 395-396, 2012.

[9] G. Bianchi, A. Detti, A. Caponi, and N. Blefari Melazzi, "Check before storing: what is the performance price of content integrity verification in lru caching?," SIGCOMM Comput. Comm. Rev., vol. 43, pp. 59-67, July 2013.

[10] E. Coffman and P. Denning, Operating Systems Theory. Englewood Cliffs (NJ): Prentice-Hall, 1973.

[11] R. Fonseca, V. Almeida, M. Crovella, and B. Abrahao, "On the intrinsic locality of web reference streams," in INFOCOM, 2003.

[12] V. Almeida, A. Bestavros, M. Crovella, and A. de Oliveira, "Characterizing reference locality in the www," in IEEE PDIS, 1996.

[13] S. Jin and A. Bestavros, "Sources and characteristics of web temporal locality," in IEEE MASCOTS, 2000.

[14] S. Traverso, M. Ahmed, M. Garetto, P. Giaccone, E. Leonardi, and S. Niccolini, "Temporal locality in today's content caching: Why it matters and how to model it," SIGCOMM Comput. Commun. Rev., vol. 43, pp. 5-12, Nov. 2013.

[15] M. Garetto, E. Leonardi, and S. Traverso, "Efficient analysis of caching strategies under dynamic content popularity," in Infocom'15, 2015.

[16] F. Olmos, B. Kauffmann, A. Simonian, and Y. Carlinet, "Catalog dynamics: Impact of content publishing and perishing on the performance of a lru cache," in ITC, pp. 1-9, IEEE, 2014.

[17] L. Breslau, P. Cao, L. Fan, G. Phillips, and S. Shenker, "Web caching and zipf-like distributions: evidence and implications," in INFOCOM, pp. 126-134, 1999.

[18] M. Cha, H. Kwak, P. Rodriguez, Y.-Y. Ahn, and S. Moon, "Analyzing the Video Popularity Characteristics of Large-Scale User Generated Content Systems," IEEE/ACM Trans. on Netw., vol. 17(5), pp. 1357-1370, 2009.

[19] C. Fricker, P. Robert, J. Roberts, and N. Sbihi, "Impact of traffic mix on caching performance in a content-centric network," in IEEE NOMEN Workshop, 2012.

[20] T. Johnson and D. Shasha, "2Q: A Low Overhead High Performance Buffer Management Replacement Algorithm," in VLDB, 1994.

[21] P. R. Jelenković and A. Radovanović, "The persistent-access-caching algorithm," Random Struct. Algorithms, vol. 33, pp. 219-251, Sept. 2008.

[22] G. Rossini and D. Rossi, "Coupling caching and forwarding: Benefits, analysis, and implementation," in ICN '14, (New York, NY, USA), pp. 127-136, ACM, 2014

[23] P. R. Jelenković and X. Kang, "Characterizing the miss sequence of the 1ru cache," SIGMETRICS Perform. Eval. Rev., vol. 36, pp. 119-121, Aug. 2008. 
[24] E. Gelenbe, "A unified approach to the evaluation of a class of replacement algorithms," IEEE Trans. Comput., vol. 22, pp. 611-618, June 1973.

[25] A. Finamore, M. Mellia, M. Meo, M. M. Munafò, and D. Rossi, "Experiences of Internet traffic monitoring with Tstat," IEEE Network, 2011.

[26] N. C. Fofack, P. Nain, G. Neglia, and D. Towsley, "Performance evaluation of hierarchical TTL-based cache networks," Computer Networks, vol. 65, pp. 212-231, 2014

[27] P. R. Jelenković, "Asymptotic approximation of the move-to-front search cost distribution and least-recently used caching fault probabilities," Annals of Applied Probability, vol. 9, no. 2, pp. 430-464, 1999.

[28] P. Jelenković and A. Radovanović, "Asymptotic insensitivity of least-recently-used caching to statistical dependency," in INFOCOM, 2003.

[29] P. R. Jelenković and A. Radovanović, "Least-recently-used caching with dependent requests," Theoretical Computer Science, vol. 326, no. 13, pp. 293-327, 2004.

[30] P. R. Jelenković and M. S. Squillante, "Critical sizing of lru caches with dependent requests," Journal of Applied probability, vol. 43, no. 4, pp. 1013-1027, 2006.

[31] E. J. O'Neil, P. E. O'Neil, and G. Weikum, “The lru-k page replacement algorithm for database disk buffering," in SIGMOD '93, (New York, NY, USA), pp. 297-306, ACM, 1993.

[32] D. Lee, J. Choi, J. H. Kim, S. H. Noh, S. L. Min, Y. Cho, and C. S. Kim, "LRFU: A spectrum of policies that subsumes the least recently used and least frequently used policies," IEEE Trans. Comput., vol. 50, pp. 1352-1361, Dec. 2001.

[33] I. Psaras, R. Clegg, R. Landa, W. Chai, and G. Pavlou, "Modelling and Evaluation of CCN-Caching Trees," in IFIP NETWORKING, vol. 6640 of Lecture Notes in Computer Science, pp. 78-91, Springer Berlin, 2011.

[34] G. Carofiglio, M. Gallo, L. Muscariello, and D. Perino, "Modeling data transfer in content-centric networking," ITC, 2011.

[35] Z. Li, G. Simon, and A. Gravey, "Caching policies for in-network caching," in ICCCN, 2012.

[36] E. Rosensweig, D. J. Menache, and J. Kurose, "On the Steady-State of Cache Networks," in INFOCOM, 2013.

[37] N. Laoutaris, H. Che, and I. Stavrakakis, "The LCD interconnection of LRU caches and its analysis," Perform. Eval., vol. 63, pp. 609-634, July 2006.

[38] N. C. Fofack, On models for performance analysis of a core cache network and power save of a wireless access network. Theses, Université Nice Sophia Antipolis, Feb. 2014.

[39] N. C. Fofack, M. Dehghan, D. Towsley, M. Badov, and D. L. Goeckel, "On the performance of general cache networks," in VALUETOOLS '14, pp. 106-113, 2014.

[40] D. Berger, P. Gland, S. Singla, and F. Ciucu, "Exact analysis of TTL cache networks," Perform. Eval., vol. 79, pp. $2-24,2014$. 